\title{
Transition Within Leeward Plane of Axisymmetric Bodies at Incidence in Supersonic Flow
}

\author{
Naoko Tokugawa ${ }^{1}$ \\ Aviation Program Group, Japan Aerospace Exploration Agency, Tokyo 181-0015, JAPAN \\ Meelan Choudhari ${ }^{2}$ \\ NASA Langley Research Center, Hampton, VA 23681, U.S.A. \\ Hiroaki Ishikawa ${ }^{3}$ \\ Sanko-Soft Co. Ltd., Tokyo, 169-0075, JAPAN \\ Yoshine Ueda ${ }^{4}$ \\ Tokyo Business Service Co. Ltd., Tokyo 160-0023, JAPAN \\ Keisuke Fujii ${ }^{5}$ and Takashi Atobe ${ }^{6}$ \\ Aerospace Research and Development Directorate, Japan Aerospace Exploration Agency, Tokyo 182-8522, JAPAN
}

Fei Li, ${ }^{7}$ Chau-Lyan Chang, ${ }^{8}$ and Jeffery White ${ }^{9}$

NASA Langley Research Center, Hampton, VA 23681, U.S.A.

\begin{abstract}
Boundary layer transition along the leeward symmetry plane of axisymmetric bodies at nonzero angle of attack in supersonic flow was investigated experimentally and numerically as part of joint research between the Japan Aerospace Exploration Agency (JAXA) and National Aeronautics and Space Administration (NASA). Transition over four axisymmetric bodies (namely, Sears-Haack body, semi-Sears-Haack body, straight cone and flared cone) with different axial pressure gradients was measured in two different facilities with different unit Reynolds numbers. The semi-Sears-Haack body and flared cone were designed at JAXA to broaden the range of axial pressure distributions. For a body shape with an adverse pressure gradient (i.e., flared cone), the experimentally measured transition patterns show an earlier transition location along the leeward symmetry plane in comparison with the neighboring azimuthal locations. For nearly zero pressure gradient (i.e.,straight cone), this feature is only observed at the larger unit Reynolds number. Later transition along the leeward plane was observed for the remaining two body shapes with a favorable pressure gradient. The observed transition patterns are only partially consistent with the numerical predictions based on linear stability analysis. Additional measurements are used in conjunction with the stability computations to explore the phenomenon of leeward line transition and the underlying transition mechanism in further detail.
\end{abstract}

\section{Nomenclature}

$C \quad=$ heat capacitance of polysulphone

$C_{p} \quad=$ surface pressure coefficient $\left(p-p_{\infty}\right) /\left(1 / 2 \rho_{\infty} U_{\infty}^{2}\right)$

$L \quad=$ model length

\footnotetext{
${ }^{1}$ Associate Senior Researcher, Supersonic Transport Team, tokugawa.naoko@jaxa.jp.

${ }^{2}$ Aerospace Technologist, Computational Aero Sciences Branch, M.S. 128. Associate Fellow, AIAA.

${ }^{3}$ Researcher, Member, AIAA.

${ }^{4}$ Researcher.

${ }^{5}$ Associate Senior Researcher, Wind Tunnel Technology Center, Member, AIAA.

${ }^{6}$ Associate Senior Researcher, Fluid Dynamics Group.

${ }^{7}$ Aerospace Technologist, Computational Aero Sciences Branch, M.S. 128.

${ }^{8}$ Aerospace Technologist, Computational Aero Sciences Branch, M.S. 128. Senior Member, AIAA.

${ }^{9}$ Aerospace Technologist, Computational Aero Sciences Branch, M.S. 128. Senior Member, AIAA.
} 


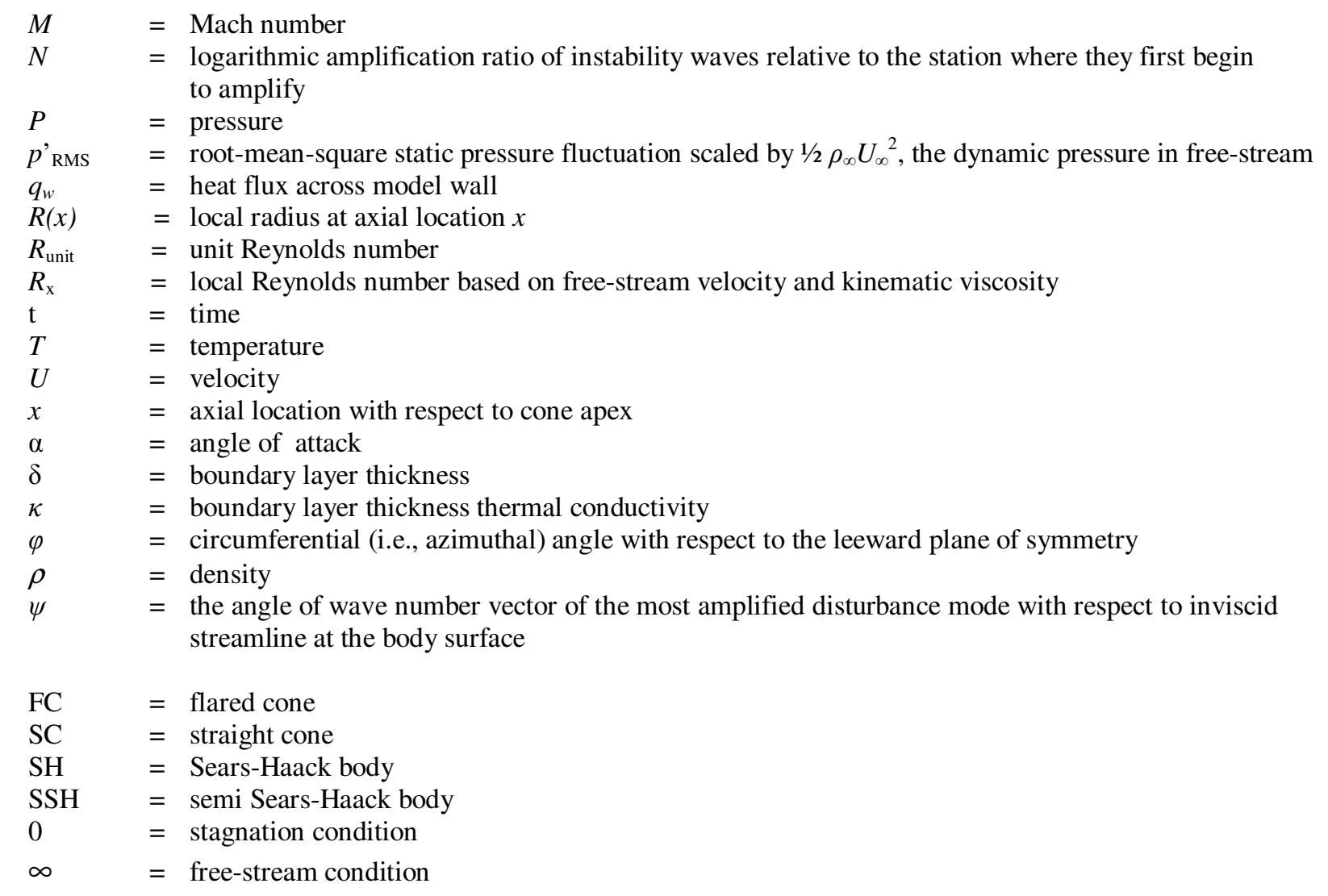

\section{Introduction}

PPLICATION of laminar flow technology to airplane lifting surfaces (i.e., wing and empennage) has been extensively investigated over the years. Viscous drag reduction for the wing and empennage surfaces leads to an increased relative contribution to the overall drag from turbulent flow over the fuselage and, in turn, increases the potential for further improvement in aerodynamic efficiency via natural laminar flow (NLF) over the fuselage surface, especially near the nose of the aircraft. Vijgen and Holmes ${ }^{1}$ argued that a laminar nose region also decreases the turbulent drag over the rest of the fuselage and has a favorable impact on the wing-fuselage interaction. According to them, a total of 30 percent laminar flow over the fuselage of an advanced, subsonic business jet aircraft can lead to nearly 7 percent reduction in the total drag. Component drag budgets for supersonic aircraft with various planform shapes ${ }^{2}$ suggest that smaller yet comparable reductions in total drag may also be expected through NLF on the fuselage nose. Despite the potential for viscous drag reduction via increased NLF over the fuselage, the pertinent experimental database is rather limited in general and almost non-existent for supersonic aircraft. As an initial step to help alleviate this deficiency, the present paper addresses the combined effects of angle-of-attack and axial pressure gradient on boundary layer transition over canonical shapes of axisymmetric bodies, with an emphasis on transition characteristics near the leeward line of symmetry.

Transition in boundary layers on axisymmetric bodies in supersonic flow has been extensively studied in the literature. Particularly noteworthy in this context are the tests on a common 5-degree half-angle cone model at zero angle of incidence in various wind tunnel facilities as well as in flight, ${ }^{3}$ and the quiet tunnel measurements of a different 5-deg cone model at zero and nonzero angles of incidence in the Mach 3.5 Supersonic Low Disturbance Tunnel at NASA Langley.,

Despite the simplicity of the body shape, supersonic flow over a straight cone with circular cross section is known to exhibit a rich transition behavior. At a zero angle of incidence $(\alpha=0)$, the boundary layer flow is axisymmetric; hence, transition at supersonic free-stream Mach numbers is dominated by first mode instability. At nonzero angles of attack, the boundary layer becomes three dimensional and the inviscid streamlines at the surface become curved due to the azimuthal pressure gradient from the windward to the leeward side. Therefore crossflow occurs and the boundary layer over the side region (i.e., in between the windward and leeward planes of symmetry) becomes 
increasingly susceptible to crossflow instability as the angle of attack is increased. At Mach 2, for instance, crossflow transition first occurs at approximately 60 degrees on either side of the leeward symmetry plane. ${ }^{6}$ Even at a finite angle of attack, supersonic boundary layer flow along the windward symmetry plane has been shown to exhibit a nearly self-similar behavior analogous to the case of zero incidence ${ }^{7}$ and, furthermore, the instability amplification within this plane has been shown to remain dominated by first mode instability.

It is well-known from early work on supersonic flow past straight cones ${ }^{7}$ that, over an intermediate range of angles of attack (i.e., $\alpha$ values that are not close to zero but are below the range where significant crossflow separation is observed:somewhat less than the cone half angle), the boundary layer flow along the leeward plane of symmetry evolves rather differently than elsewhere on the cone surface. Specifically, the convergence of low-speed secondary flow from both sides of the leeward symmetry plane leads to a lift-up effect within the plane of symmetry, and hence to a significant thickening of the boundary layer along the leeward plane. The thicker boundary layer profiles exhibit a strong inflectional behavior, and hence are more unstable than the boundary layer flow in the adjoining region of the cone.

Preliminary computations performed at the beginning of this effort showed that the boundary layer profiles along the leeward symmetry plane are highly sensitive to the magnitude of the axial pressure gradient. When the pressure gradient along the leeward ray is favorable, such as for the flow past the Sears-Haack body at a small angle of attack, the lift-up effect within the leeward symmetry plane is substantially reduced. Essentially, the acceleration of the streamwise velocity component enables the flow to carry the low-speed fluid converging from both sides of the leeward plane. Consequently, the velocity profiles along the leeward ray can remain non-inflectional over longer distances, resulting in a more stable boundary layer flow. This alters the relative locations of transition location along the leeward plane and the earliest location of crossflow-induced transition over the side of the cone. Indeed, major changes in the transition front characteristics can occur as the body shape is varied. An understanding of these changes is relevant to the aerodynamic design of an aircraft nose targeting a longer region of NLF.

Transition fronts with three local minima, one along the leeward ray and one each due to crossflow transition on either side have previously been observed and/or predicted in the context of straight cones ${ }^{6,8-10}$ and a delta wing configuration. ${ }^{11}$ However, the physics of transition along the leeward plane and the effect of streamwise pressure gradient on the corresponding transition location has not been scrutinized in detail, perhaps due to the narrow width of the transition lobe centered on the leeward ray and/or the low wall shear associated with the thicker boundary layer in that region. The latter factors aside, the ubiquitous nature of analogous transition patterns in the context of fully $3 \mathrm{D}$ high-speed flows over slender bodies ${ }^{12,13}$ makes it even more useful to examine the transition process along the leeward symmetry plane in greater detail.

To that end, boundary layer transition along the leeward ray of axisymmetric bodies at a nonzero angle of attack in supersonic flow was investigated both experimentally and numerically as part of joint research between JAXA and NASA. The objective of this research program is to improve the knowledge base for transition mechanisms relevant to the nose region of the fuselage. More in-depth computational analysis with an emphasis on transition behavior adjacent to and away from the leeward ray will be described in a separate paper. Another complementary component of the joint research involves quiet tunnel measurements and coordinated computations for a 7-degree circular cone at both zero and non-zero angles of incidence in NASA's Mach 3.5 Supersonic Low Disturbance Tunnel. ${ }^{14}$

The following section introduces the four different axisymmetric bodies with varying axial pressure gradients, which were used during the present investigation. Experimental measurements of transition are described in Section III. Numerical results pertaining to both the mean velocity profiles along the leeward ray and the corresponding linear stability characteristics are described in Section IV. More in-depth comparison between experimental measurements and the numerical predictions is outlined in Section V. Finally, a summary of the present work and concluding remarks are presented in Section VI.

\section{Model Geometry and Flow Conditions}

The four different axisymmetric bodies targeted in the present investigation are the Sears-Haack body, a semiSears-Haack body, a straight cone, and a flared cone. The shapes of all four bodies are plotted in Fig. 1, wherein $x$ denotes the axial coordinate relative to the cone apex and $R$ represents the local body radius at a given station.

The Sears-Haack body (abbreviated as SH in the following) produces the least wave drag for a given length and maximum diameter based on slender body theory (i.e., solution of the linearized potential equation). Its shape is defined by the following expression for the streamwise distribution of local radius $R_{\mathrm{SH}}(x)$ :

$$
R_{\mathrm{SH}}(x)=A_{0}\left[\left(x / L_{\mathrm{SH}}\right)\left\{1-\left(x / L_{\mathrm{SH}}\right)\right\}\right]^{3 / 4},
$$

where $L_{\mathrm{SH}}=1.194938 \mathrm{~m}$ and $A_{0}=0.09657 \mathrm{~m}$ for cases SH-2deg-99 and SH-2deg-70 from Table 1 . 
The semi-Sears-Haack body (abbreviated as SSH in the following) corresponds to a linearly weighted mean of the radius distributions for the Sears-Haack body and straight cone, as expressed by the radius distribution:

$$
R_{\mathrm{SSH}}(x)=0.3 R_{\mathrm{SH}}(x)+0.7 R_{\mathrm{SC}}(x),
$$

where $R_{S C}(x)$ corresponds to the local radius of the straight cone as defined below.

The straight cone (abbreviated as SC in the following) geometry is defined by the cone half-angle $\Theta$, which is equal to 5 degrees for the present study. The variation of model radius with the axial coordinate is defined as follows:

$$
R_{\mathrm{SC}}(x)=x \tan \Theta .
$$

Two separate straight cone models were used in the experiments. The first model, denoted as SC1, had an axial length of $L=700 \mathrm{~mm}$, whereas the other, shorter model (SC2) had an axial length of $L=300 \mathrm{~mm}$.

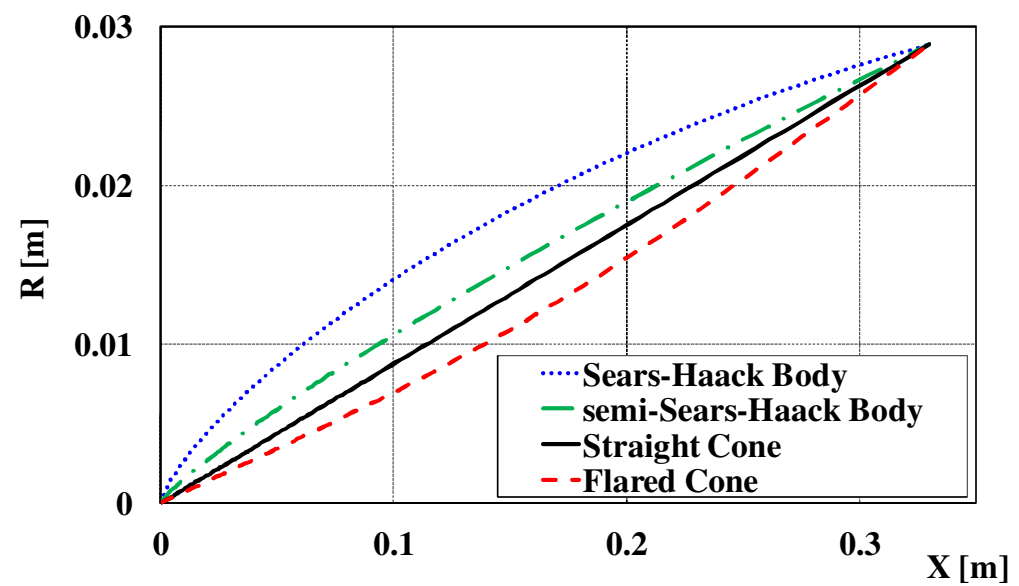

Figure 1. Geometry of selected axisymmetric shapes. Both abscissa and ordinate are in meters.

\begin{tabular}{|c|c|c|c|}
\hline \multicolumn{2}{|l|}{ Geometric Configuration } & \multirow{2}{*}{\multicolumn{2}{|c|}{$\begin{array}{c}\text { Flow Condition } \\
M_{\infty}=2\end{array}$}} \\
\hline \multirow{4}{*}{ Shape } & \multirow{4}{*}{$\begin{array}{l}\text { Angle of Attack } \\
\text { (degrees) }\end{array}$} & & \\
\hline & & $\begin{array}{c}P_{0}=70 \mathrm{kPa} \\
T_{\infty}=186 \mathrm{~K} \\
T_{w} / T_{a d}=1.0 \\
\end{array}$ & $\begin{array}{c}P_{0}=99 \mathrm{kPa} \\
T_{\infty}=165 \mathrm{~K} \\
T_{w} / T_{a d} \approx 1.08\end{array}$ \\
\hline & & $R_{\text {unit }} / \mathrm{m} \approx 7.3 \times 10^{6}$ & $R_{\text {unit }} / \mathrm{m}=12.2 \times 10^{6}$ \\
\hline & & \multicolumn{2}{|c|}{ Case Notation } \\
\hline $\begin{array}{l}\text { Sears-Haack body }(\mathrm{SH}) \\
(L=330 \mathrm{~mm})\end{array}$ & \multirow{2}{*}{2.0} & SH-2deg-70 & SH-2deg-99 \\
\hline $\begin{array}{l}\text { Semi-Sears-Haack body (SSH) } \\
\qquad(L=330 \mathrm{~mm})\end{array}$ & & SSH-2deg-70 & SSH-2deg-99 \\
\hline \multirow{2}{*}{$\begin{array}{l}\text { Straight cone (SC) with 5-degree half angle } \\
(L=700 \mathrm{~mm} \text { for SC1, } L=300 \mathrm{~mm} \text { for SC } 2)\end{array}$} & 0.0 & & SC1-0deg-99 \\
\hline & 2.0 & SC2-2deg-70 & SC1-2deg-99 \\
\hline $\begin{array}{l}\text { Flared Cone }(\mathrm{FC}) \\
\qquad(L=330 \mathrm{~mm})\end{array}$ & 2.0 & FC-2deg-70 & FC-2deg-99 \\
\hline
\end{tabular}

Table 1: Summary of flow conditions and case notation 
Finally, the flared cone (abbreviated as FC in the following) geometry is defined by the following distribution of model radius:

$$
R_{\mathrm{FC}}(x)=-1.0478 \times 10^{-9} x^{4}+6.9293 \times 10^{-7} x^{3}-6.1497 \times 10^{-5} x^{2}+6.998 \times 10^{-2} x-6.2485 \times 10^{-4},
$$

where the axial coordinate $x$ is measured in meters.

The semi-Sears-Haack body and flared cone configuration were originally designed in JAXA for investigating the influence of pressure gradient on the flow characteristics near the leeward symmetry plane. ${ }^{15}$ As mentioned in the Introduction, the effects of secondary flow convergence from both sides of the leeward symmetry plane and the associated lift-up of low-speed fluid away from the surface are expected to increase as the axial flow along this plane goes from accelerated ( $\mathrm{SH}, \mathrm{SSH})$ to nearly constant velocity along the cone axis (SC) to decelerated (FC).

Table 1 provides a summary of the relevant test cases as well as introducing a composite notation that combines the information about the experimental model, angle-of-attack, and tunnel stagnation pressure. For example, the case SC1-0deg-99 from Table 1 refers to the straight cone model SC1 at 0-degree incidence and a tunnel stagnation pressure of $99 \mathrm{kPa}$.

\section{Experimental Measurements}

\section{A. Wind Tunnel Facilities}

The wind tunnel experiments were conducted by JAXA in two different facilities, with the lead author of this paper as the principal investigator. The measurements in each facility provided information about the transition front over the model as well as the static pressure fluctuations in the free stream. The first facility was the $0.2 \mathrm{~m} \times 0.2 \mathrm{~m}$ supersonic Wind Tunnel (SWT2) in JAXA. ${ }^{16}$ The SWT2 is a continuously running tunnel that allows independent and continuous variations in both Mach number $\left(1.5<M_{\infty}<2.5\right)$ and total pressure $\left(55 \mathrm{kPa}<P_{0}<100 \mathrm{kPa}\right)$. The nominally controlled total flow temperature $T_{0}$ equals $335 \mathrm{~K}$, but can be increased if necessary by terminating the supply of the cooling water. For the work described in this paper, the flow conditions for all tests in SWT2 were fixed at $M_{\infty}=2, P_{0}=70 \mathrm{kPa}$, and $T_{0}=335 \mathrm{~K}$. The second facility used in the present work was the $0.6 \mathrm{~m} \times 0.6 \mathrm{~m}$ High Speed Wind Tunnel at Fuji Heavy Industries in Japan, which is an in-draft type facility. It will be denoted as FWT in this paper. The freestream Mach number in FWT can be varied in steps, and was maintained at $M_{\infty}=2$ for the experiments reported herein. The total pressure $P_{0}$ and total temperature $T_{0}$ are almost atmospheric but cannot be prescribed. Therefore the $P_{0}$ and $T_{0}$ values vary from run to run. However the magnitudes of these variations are small and result in a less than 5\% variation in the unit Reynolds number. For transition measurements, the original Schlieren window in both wind tunnels was replaced by a sapphire-glass window to enable the measurement of model surface temperature using an infrared (IR) camera.

Neither SWT2 nor FWT incorporate any form of boundary layer control to delay transition in the nozzle wall boundary layer. Hence, as in other conventional supersonic facilities, the tunnel disturbance environment is expected to include a substantial component due to the acoustic radiation from the turbulent boundary layers along the tunnel wall. Somewhat surprisingly, however, the free-stream static pressure fluctuations in both facilities have been found to be rather low at the test Mach number of $M_{\infty}=2\left(p^{\prime}{ }_{\text {RMS }}<0.1 \%\right.$ in SWT2 and $p^{\prime}{ }_{\text {RMS }}=0.04 \%$ for FWT). Here, $p$ ' ${ }_{\text {RMS }}$ denotes the root-mean-square value of the surface pressure fluctuation scaled by the dynamic pressure of the free-stream and was measured using a Kulite pressure transducer mounted on a straight cone model at zero angle of attack. Given the low amplitudes of free-stream disturbances, both facilities were deemed acceptable, at least for studying the mechanisms for transition within the boundary layer flow along the leeward plane. As an independent a posteriori assessment of the tunnel disturbance environment, the transition Reynolds numbers for axisymmetric flow past a straight cone in SWT2 and FWT were compared with those in other conventional and low disturbance (i.e., quiet) wind tunnel facilities as well as those measured in a flight experiment. This comparison indicated that the transition behavior in SWT2 and FWT is effectively similar to that in other conventional wind tunnels in spite of the lower levels of free-stream pressure fluctuations in these two facilities.

\section{B. Test Models}

The geometric specifications of the four test models used for transition measurements were summarized in Table 1. Each model was made of polysulphone (PSU) with a stainless steel core. Since the polysulphone resin was not suitable to process the sharp apex, the latter was made of stainless steel. The aft end of the cone that connects with the model sting was also made of stainless steel. Due care was taken to avoid any surface height discontinuities at the juncture between the nose and the main part of cone. The SC1 straight cone model was longer, with a total axial length of $L=700 \mathrm{~mm}$. The length of the SC2 straight cone model was $300 \mathrm{~mm}$ and the length of all of the other 
models was $330 \mathrm{~mm}$. All models had a $\beta$-sting function in the form of a hinge that enabled the cone to be set at a nonzero yaw angle if necessary. The $\beta$-sting function permitted the observation of leeward and windward views from the side. To measure the surface pressure fluctuation $p_{\text {RMS }}$, a Kulite sensor was flush-mounted at $x=170 \mathrm{~mm}$.

The apex shape was examined through micrographs and was found to have significant azimuthal variations. The apex diameter is defined as the diameter of a circular fit to the apex shape, averaged over photographs taken from four different directions. The apex diameter determined in this manner was found to be different for each model, with the overall range being $0.041 \mathrm{~mm}$ to $0.260 \mathrm{~mm}$.

The surface roughness was estimated by reproducing the roughness pattern on small samples of dental resin (Pattern Resin 1-1PKG). The resin was hardened together with a paper backing that could be easily peeled off with the help of a hole at the center of the paper. The reproduced roughness on the resin sample was measured with a laser displacement sensor (LT-8010; Keyence). The roughness amplitudes (measured as arithmetic mean of roughness heights) for all four models were nearly the same, approximately in the range of $0.6 \mu \mathrm{m} \sim 0.7 \mu \mathrm{m}$.

The effects of azimuthal variations near the cone apex and the surface roughness on either the mean flow or the disturbance evolution within the downstream region are not addressed in this paper. Thus, all computations as reported in sections IV and V, respectively, are performed for the design configurations as shown earlier in Fig. 1.

\section{Instrumentation and Measurement Methodologies}

Since the rate of heat transfer reflects the transport properties of the boundary layer flow, the transition front can be inferred as the locus of points associated with a rapid streamwise gradient in surface heat transfer. The surface temperature distribution was measured using an infra-red camera (TVS-8502, Nippon Avionics Co. Ltd.), and subsequently converted to a surface heat flux distribution under the assumption of semi-infinite, one-dimensional thermal conduction through a uniform medium with temperature invariant thermal properties. The heat transfer rate as a function of time function has been calculated according the following equation ${ }^{17}$ :

$$
q_{w}(t)=\sqrt{\frac{\rho C \kappa}{\pi}}\left\{\frac{T(t)}{\sqrt{t}}+\int_{0}^{t} \frac{T(t)-T(\tau)}{(t-\tau)^{3 / 2}} d \tau\right\} .
$$

Because of the relatively low thermal diffusivity of the insulative material of the model, the Fourier number $\left(=\kappa t / \rho C R^{2}\right)$ based on the thickness (i.e. the local model radius) of the model remained less than 0.04 over the duration of the IR measurement. These low values support the validity of the assumption of semi-infinite model thickness during the present experiments. For the tests conducted in FWT, the initial surface temperature distribution can be reasonably assumed to be uniform. However, because of the long duration of the tests conducted in the closed circuit wind tunnel SWT2, the temperature distribution at the model surface has nearly reached an equilibrium at the start of the IR measurement and, therefore, the surface heat flux is very small. To obtain a measurable heat flux, a small but sudden, i.e., step-like change was applied to the SWT2 stagnation temperature $\left(\Delta T_{0} \approx 5 \mathrm{~K}\right)$ after reaching the equilibrium temperature distribution for $T_{0} \sim 335 \mathrm{~K}$. The 5 degrees variation in stagnation temperature is negligible from the standpoint of comparison with the numerical prediction, since it corresponds to just $2 \%$ variation in the unit Reynolds number. The measured changes in surface temperature in response to the change in $T_{0}$ were used in conjunction with Eq. 5 to estimate the heat transfer rates during the SWT2 tests. The initial temperature in this case was based on the meaured distribution of equilibrium temperature before the step-like change in stagnation temperature was applied. An analogous technique for IR-based transition measurements was used during a recent flight experiment involving crossflow transition at a low subsonic Mach number. ${ }^{18}$ The heat transfer distributions inferred via the procedure outlined above are shown in Fig. 2, where the transition front is clearly marked by a sharp, negative streamwise gradient in the images corresponding to the measurements in FWT and by a sharp positive gradient for the images acquired in the closed circuit tunnel SWT2. The reason behind the opposite gradients in heat transfer distributions in the two facilities is clarified in subsection D below.

Phosphor thermography and temperature-sensitive-paint (TSP) techniques are commonly utilized for transition detection in high-speed boundary layers. However, compared to these techniques, measurements using an IR camera have the advantage that it does not affect the smoothness of the measurement surface. Maintaining the surface finish is particularly important for the present study because of the potential significance of stationary crossflow instability for transition over the side region of the cone models (i.e., away from the symmetry planes). The stationary crossflow modes can be easily excited by surface roughness, such as that introduced by the application of the phosphor coating or the TSP. Hence, the latter two measurement techniques can artificially influence the natural transition process over the body surface and thus were not used during the current study.

Surface pressure fluctuations on the SC2 and FC models were measured by a single Kulite sensor (XCS-062), which was flush-mounted at the axial location of $x=170 \mathrm{~mm}$ on each of those models. The sensor size corresponds 
to a diameter of $1.6 \mathrm{~mm}$. The acquired signal was analyzed using a 16-bit multi-functional digital FFT analyzer (CF-5210; ONO-SOKKI).

\section{Pattern of heat transfer distribution}

Heat transfer maps for the test conditions of interest are shown in Figures 2(a) through 2(h). The pair of white arrows in each of these figures indicates the lateral boundary of the region of IR measurement and the two black arrows indicate the edge of the wind tunnel model. The yellow arrows indicate the location of an aluminum tape, which was used as a fiduciary mark along the rear section of the model. A thin black transverse arrow is used in each figure (with the exception of Fig. 2(c)) to indicate a reference location along the model axis. The absence of the thin black transverse arrow in Fig. 2(c) is due to the low accuracy of the reference aluminum tape for the SSH2deg-99 case.

It may be noted that the heat transfer distributions at $P_{0}=70 \mathrm{kPa}$ and $P_{0}=99 \mathrm{kPa}$ have opposite signs. The data at $P_{0}=70 \mathrm{kPa}$ was acquired in SWT2 by terminating the cooling water to apply a step change in the stagnation temperature of the flow. As a result, the direction of heat transfer is from the flow to the model surface (i.e., $q_{w}>0$ ). On the other hand, the model temperature for the $P_{0}=99 \mathrm{kPa}$ runs in the short duration facility FWT is higher than the adiabatic surface temperature and, therefore, the flow cools the model, i.e., $q_{w}<0$. Nevertheless, in both cases, the transition location corresponds to a rapid rise in the magnitude of the surface heat flux.

For the SH body, transition at $P_{0}=99 \mathrm{kPa}$ is first seen to occur over the side of the cone, i.e., away from the leeward plane of symmetry (Fig. 2(a)). There is actually a local maximum in the transition location along the leeward ray, so that the overall shape of the transition front on the unrolled surface of the SH body (such that the leeward ray is located along the center) resembles a "W" shape that has been rotated anticlockwise by 90 degrees. Henceforth, this shape will be referred to as the "sideways W" pattern. The upstream transition over the side region is believed to have been caused by crossflow instabilities in that region. The shape of this crossflow transition lobe is analogous to that seen previously during quiet tunnel measurements of a Mach 3.5 delta-wing configuration as well as in the course of conventional facility measurements for a yawed circular cone at Mach 6. The cause of transition along the leeward ray (where the crossflow velocity is identically zero because of symmetry) is not immediately obvious. It could have been initiated by the amplification of linear instabilities of the laminar flow in its vicinity or induced by turbulent contamination from the adjacent region. Fig. 2(b) shows that in the lower Reynolds number case SH-2deg-70, the boundary layer flow remained laminar over the entire body surface. Figs. 2(c) and 2(d) reveal that, similar to the SH configuration, the SSH body shape also exhibits the "sideways W" shaped transition pattern, with an earlier transition location away from the leeward ray. The main difference between the transition patterns over the two bodies is that for SSH, transition is observed to occur even in the lower Reynolds number case (Fig. 2 d)).

The high Reynolds number case SC-2deg-99 (Fig. 2(e)) for the straight cone configuration indicates a qualitative change from the transition pattern observed for the SH and SSH body shapes. The transition front in this case includes an additional, narrow lobe centered on the leeward ray, indicating a different cause of transition than the crossflow instability that dominates the outer two lobes within the transition front. Because the earliest transition location within the center lobe occurs along the leeward ray, one may reasonably assume that the underlying cause of transition is the amplification of instabilities along the leeward ray. It is seen, however, that the transition location along the leeward ray is still significantly farther downstream from the earliest onset of transition within the side region. This may be due to the fact that for $\alpha=2$ degrees, the secondary flow leading to the thickening of the leeward plane boundary layer is still relatively weak. Consistent with this conjecture, Fig. 2(f) for case SC-2deg-70 shows that the center lobe disappears when the unit Reynolds number is decreased and one recovers the "sideways W" shaped transition pattern discussed earlier for the SSH and SH bodies.

The three lobed transition front observed in the higher Reynolds number straight cone case (SC-2deg-99) is also observed in both high and low Reynolds number cases involving the flared cone (Figs. 2(g-(h)). Furthermore, the transition front for both FC cases indicates a marked reversal in the relative streamwise positions of the center lobe and the outer lobes respectively, in comparison with the high Reynolds number straight cone case discussed earlier (Fig. 2(e)). In particular, the initial onset of transition along the center lobe is now significantly upstream in comparison the transition location anywhere along the outer lobes. Overall, the transition front images from Fig. 2 indicate a progressively stronger transition mechanism along the leeward ray as the axial pressure gradient becomes increasingly less favorable in going from the SH body (modestly favorable) to FC (modestly adverse).

\section{E. Extraction of transition location}

The heat transfer maps from the previous subsection outlined the qualitative nature of transition patterns over the canonical nose configurations of interest. The present subsection describes quantitative estimates of the transition 
(a) SH-2deg-99

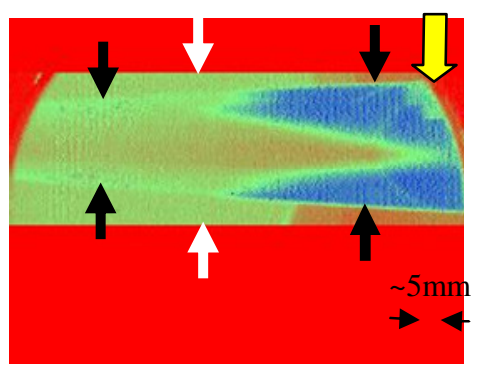

$-258.2$

(c) $\mathrm{SSH}-2$ deg-99

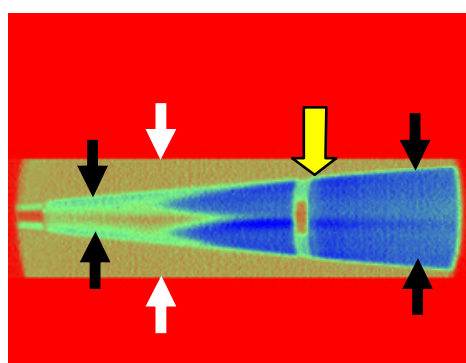

$-258.2$

$\mathrm{W} / \mathrm{m}^{2}$

(e) SC-2deg-99

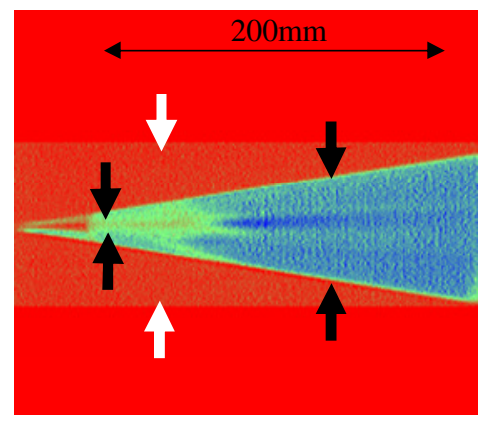

$-1000$

$\mathrm{W} / \mathrm{m}^{2}$

(g) FC-2deg-99

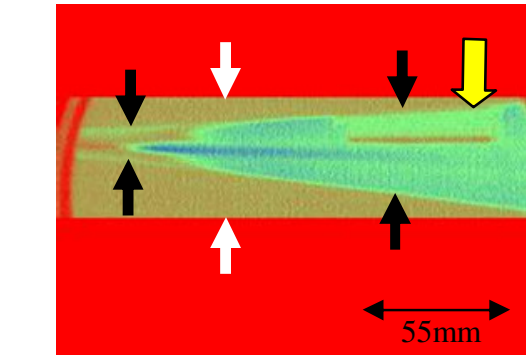

$$
\begin{array}{lll}
-182.6 & W
\end{array} \mathrm{~m}^{2} \quad 0
$$

(b) $\mathrm{SH}-2 \mathrm{deg}-70$
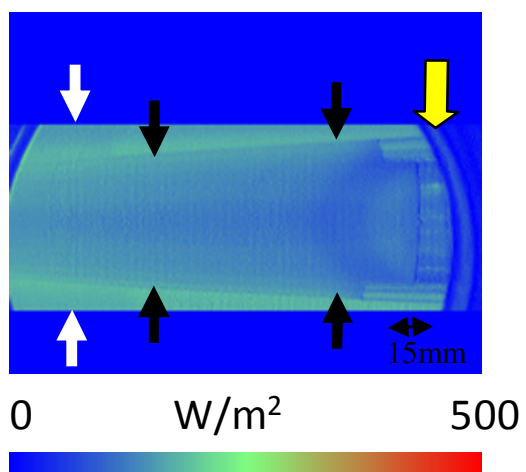

(d) $\mathrm{SSH}-2$ deg-70

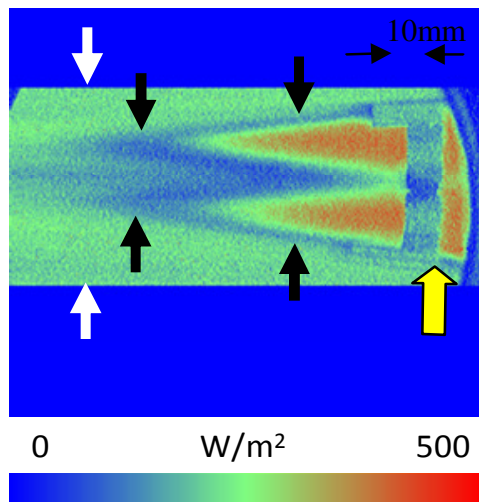

(f) SC-2deg-70

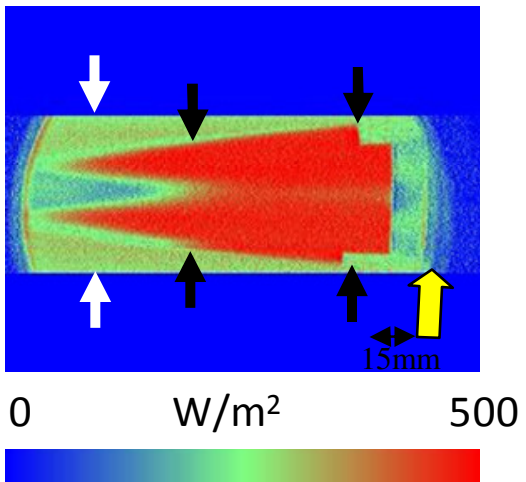

(h) FC-2deg-70

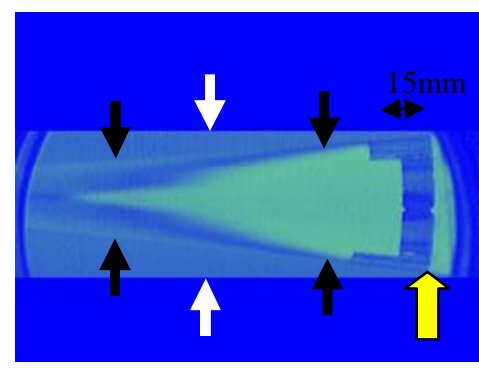

Figure 2. Heat transfer distributions for test conditions of interest (top view, approximately centered on the leeward symmetry plane). 


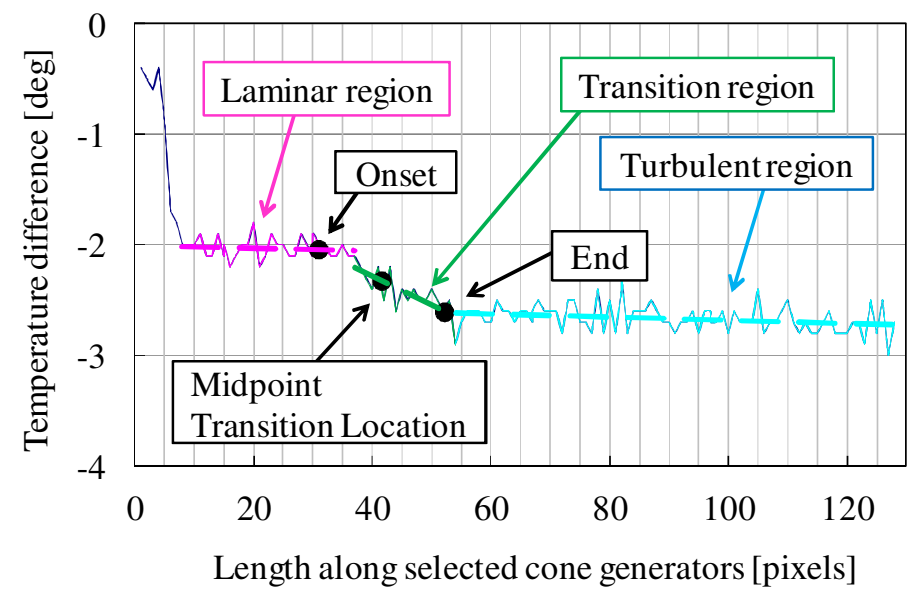

Figure 3. Sample variation of temperature difference along a iso-azimuthal angle line ( $\varphi=-20 \mathrm{deg})$.

(a) Transition onset front superposed on surface map of $\Delta T$

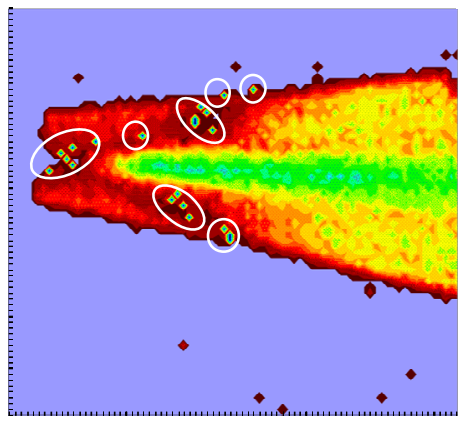

(b) Similar to (a), but mid-point of transition zone

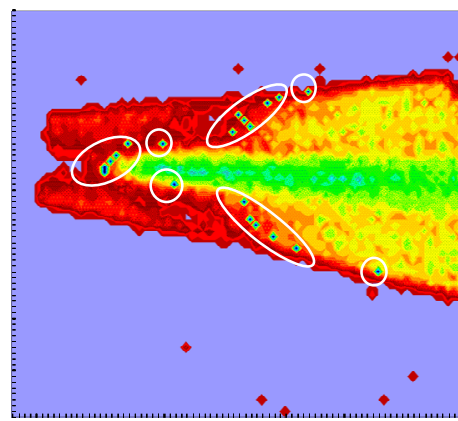

(c) Similar to (a), but end of transition zone

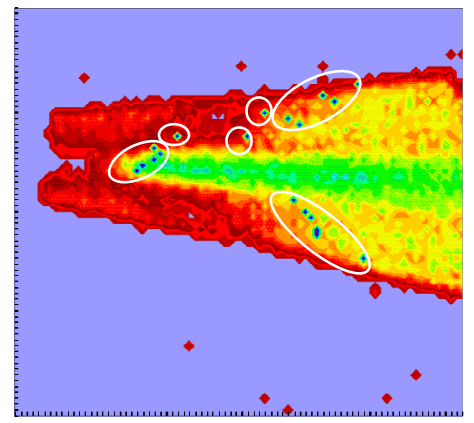

$\Delta T[\mathrm{deg}]$

- $-1.8--1.7$ - $-1.9--1.8$

$-2--1.9$

$-2.1--2$

$-2.2--2.1$

I $-2.3--2.2$

$-2.4--2.3$

$-2.5-2.4$

III. $-2.6--2.5$

$-2.7--2.6$

$\mathrm{X}-2.8--2.7$

$-2.9--2.8$

III $-3--2.9$

$-3.1--3$

$-3.2--3.1$

(1) $-3.3--3.2$

$-3.4--3.3$

ㅍ. $-3.5--3.4$

$-3.6--3.5$

$-3.7--3.6$

$-3.8--3.7$

- $-3.9--3.8$

ㄷ-4--3.9

(d) Comparison of transition fronts based on different criteria (blue dots enclosed in white circles denote transition locations along selected generators of the cone surface), along with ratios of respective transition locations

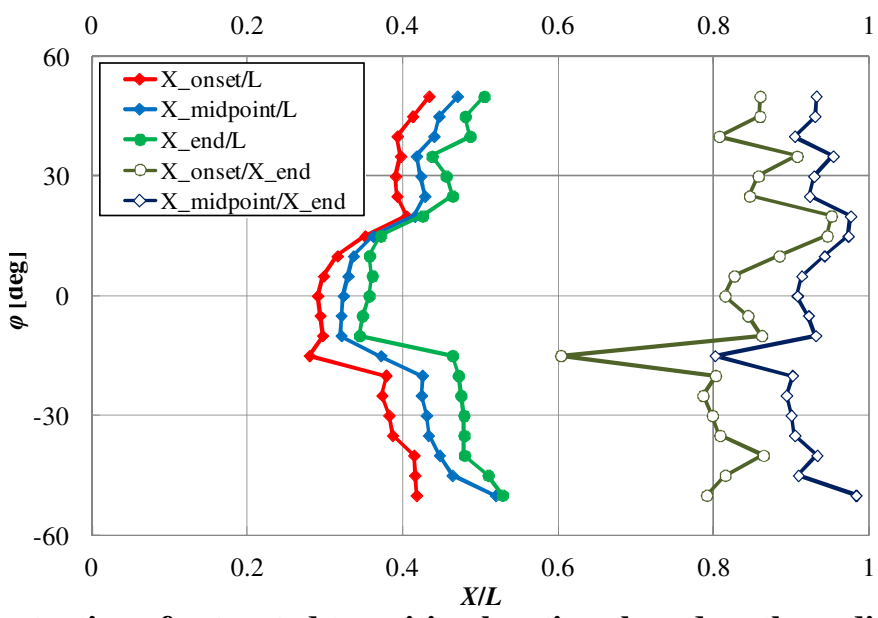

Figure 4. Illustration of extracted transition locations based on three different criteria (Configuration FC-2deg-99).

American Institute of Aeronautics and Astronautics 
front in the relevant cases. The transition location was extracted from the maps of "temperature difference $(\Delta T)$ " between two selected instants of time for a given run. For the short duration tests carried out in FWT, the two selected times correspond, respectively, to the time $t=t_{1}$ when the flow condition was stabilized in FWT and some later instant $t=t_{2}\left(t_{2}>t_{1}\right)$ when a measurable temperature difference had been established with respect to $t=t_{1}$. The corresponding times for the tests in SWT2 are based on the beginning and the end of the surface temperature response to the step-like change in stagnation temperature.

Axial variations in $\Delta T$ along selected cone generators (corresponding to lines of constant azimuthal angle along the cone surface) are extracted from the surface maps of $\Delta T$ using the location of an aluminum tape as a reference as mentioned previously. For each curve of this type, the transition region and the laminar and turbulent flow regions immediately upstream and downstream of the transition region are each approximated by a linear segment (Fig. 3). The point of intersection between the laminar and transitional segments corresponds to the onset of transition, whereas the point of intersection between the transitional and turbulent segments defines the end of transition. Midpoint of the straight line segment connecting the onset- and end-of-transition locations defines the mid-point of the transition zone.

An illustration of the transition fronts extracted using the above method is shown in Fig. 4, which corresponds to the FC-2deg-99 configuration. The blue dots enclosed within white circles in Figs. 4(a) through 4(c) correspond to the inferred loci for the beginning, mid-point, and end of transition, respectively. The locations of these blue dots are extracted from the axial variations in $\Delta T$ along selected cone generators. The shift in blue dot locations relative to the base map of $\Delta T$ indicates the progression from the beginning (Fig. 4(a)) to the end of transition (Fig. 4(c)). For comparison with transition predictions based on $\mathrm{N}$-factor methods, the locus of transition onset (i.e., beginning of transition) is more important than the other two loci. However, the primary goal behind these experiments was to understand the transition charateristics in the vicinity of the leeward plane and not to enable an accurate comparisons with numerically predicted $\mathrm{N}$-factors. With the primary objective in mind, it is both convenient and robust to define the transition front using the mid-point location, which may be visually associated with the middle of the region of highest axial gradient in the surface map of $\Delta T$. Accordingly, the midpoint locus is used as the measured transition location in the remaining part of this paper.

Fig. 4(d) indicates that the transition fronts based on the three different measures tend to be parallel to each other. The ratios of onset to end locations, and mid-point to end location as a function of the azimuthal angle are also plotted in Fig. 4(d). The average ratio of the onset to end locations is approximately 0.85 , and the ratio of midpoint to end location is approximately 0.92 . These ratios are close to those obtained during the Fisher-Dougherty flight experiment, ${ }^{3}$ with the exception of the $\varphi=-15 \mathrm{deg}$ meridian for which the above ratios have rather low values. The results in Fig. 4 also imply that the transition onset is less than $10 \%$ upstream from the midpoint of the transition zone, at least in this particular case (FC-2deg-99).

Transition fronts for the 9 cases from Table 1 are shown in Figs. 5(a)-(i). The light brown circles in these figures indicate the transition front based on the top view of the model, whereas the the dark brown circles indicate the transition front inferred from the side view of the model under the same flow conditions. There are clear but modest differences between the transition fronts inferred from the two views of each model. These differences reflect the uncertainty in mapping the IR image to surface coordinates. The factors contributing to this uncertainty include: curvature of model surface, possible inaccuracy in the location(s) of the aluminum tape used as a fiduciary mark, and inadequate resolution of the IR images. It may be noted that transition fronts for the SH-2deg-70 and SSH-2deg-99 cases were not extracted, since transition did not occur for SH-2deg-70 case and the accuracy of the reference aluminum tape was rather low for SSH-2deg-99 case. On the other hand, the transition location is not extracted in the leeward ray region for SSH-2deg-70 case, since it occurs very far downstream and cannot be extracted from the IR measurement. The open diamond and circle in Figs. 5(a)-(i) correspond, respectively, to transition locations along the leeward ray and the minimum of the crossflow transition lobe on the side, as described below.

Next, two separate scalar measures of the overall transition front are extracted from the results shown in Fig. 5. These two measures correspond, respectively, to the transition location on the leeward ray and the earliest location of the side lobe associated with crossflow transition. The values of both measures for each of the relevant cases are summarized in Table 2. Because of the previously mentioned discrepancies between transition fronts based on the side and top views, the values of the scalar measures are averaged over the two views as necessary. The transition locations for the FC-2deg-99 and FC-2deg-70 configurations are monotonically increasing functions of the azimuthal angle $\varphi$ from the leeward ray and, hence, the minimum of the side lobe could not be identified. Therefore, the location at $\varphi=30$ degrees is listed in the Table. The transition front measures extracted in this manner are are shown in Figs.5(a)-(i) by a large black open diamond and circle, respectively. (Fig. 5(j) corresponds to purely 
(a) SH-2deg-99

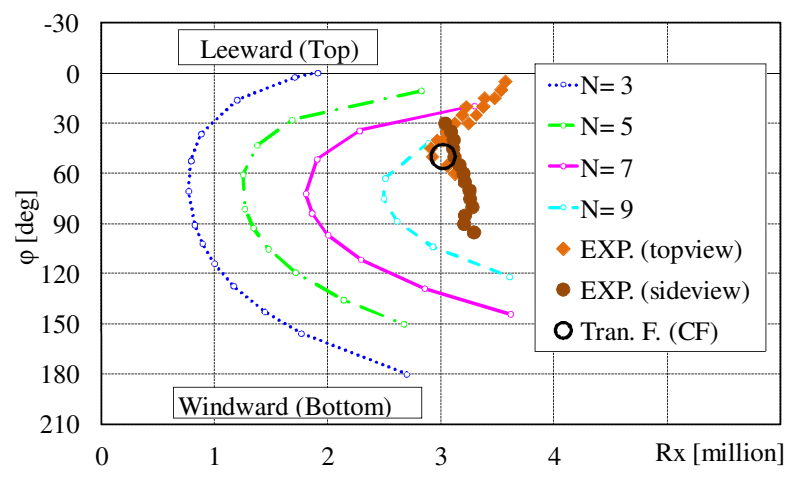

(c) SSH-2deg-99

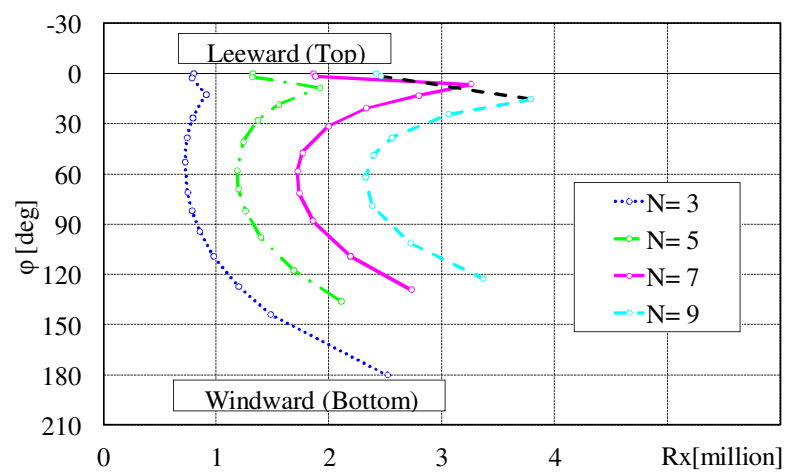

(e) SC-2deg-99

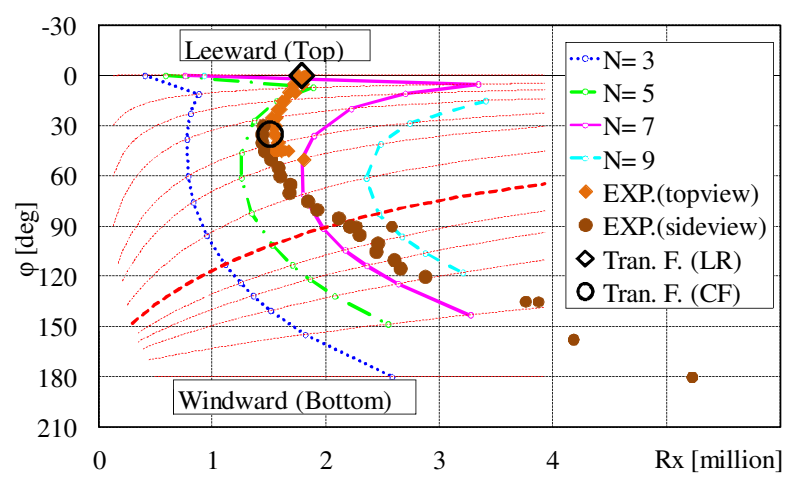

(g) FC-2deg-99

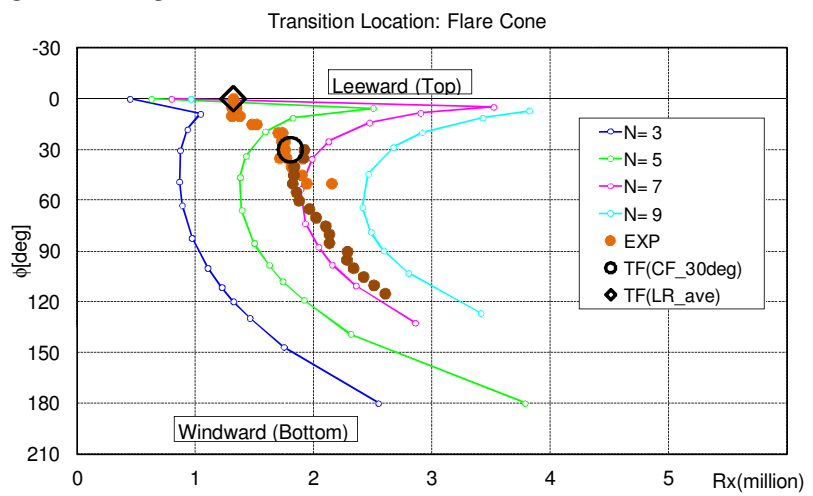

(b) $\mathrm{SH}-2$ deg-70

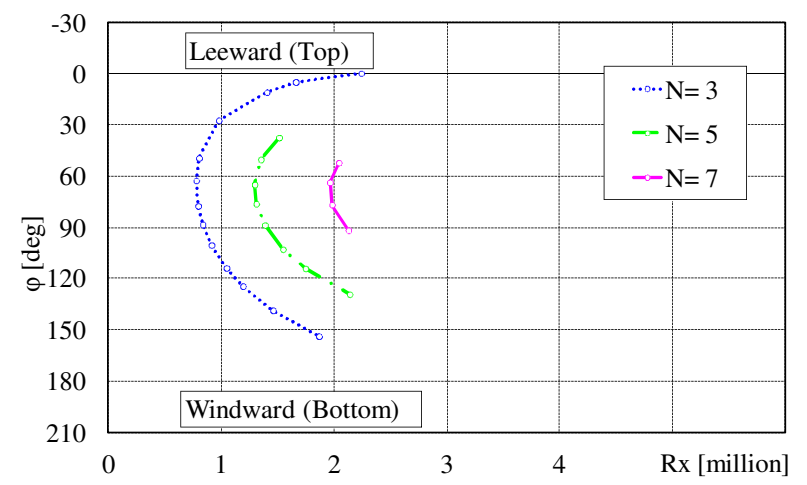

(d) $\mathrm{SSH}-2 \mathrm{deg}-70$

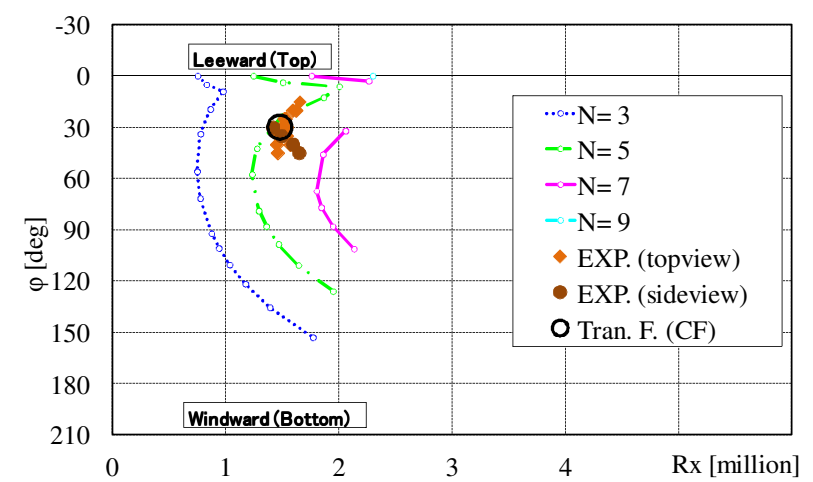

(f) SC-2deg-70

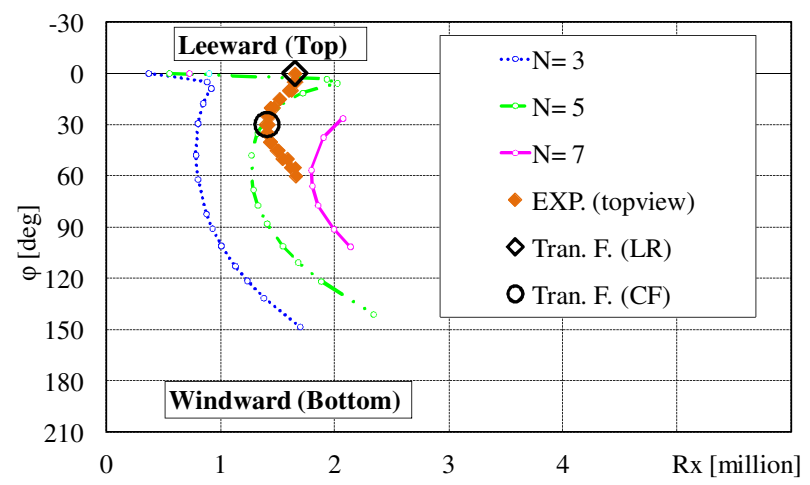

(h) FC-2deg-70

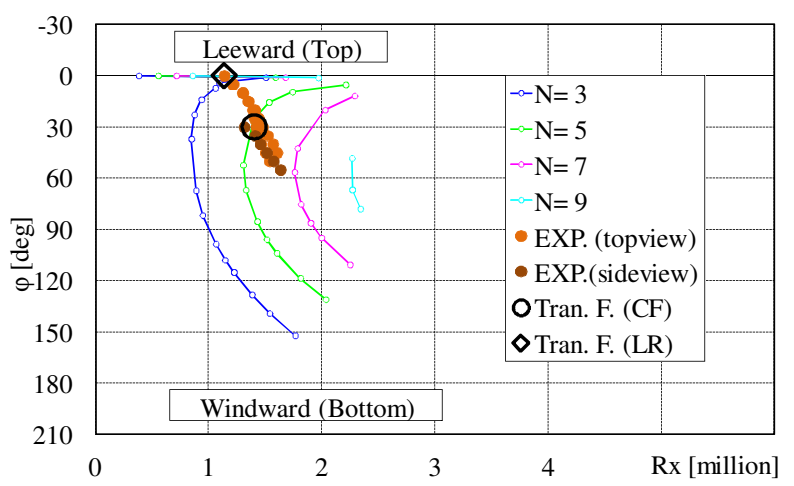

Figure 5. $N$-factor contours based on envelope method for selected test conditions.

11

American Institute of Aeronautics and Astronautics 
(i) SC-0deg-99

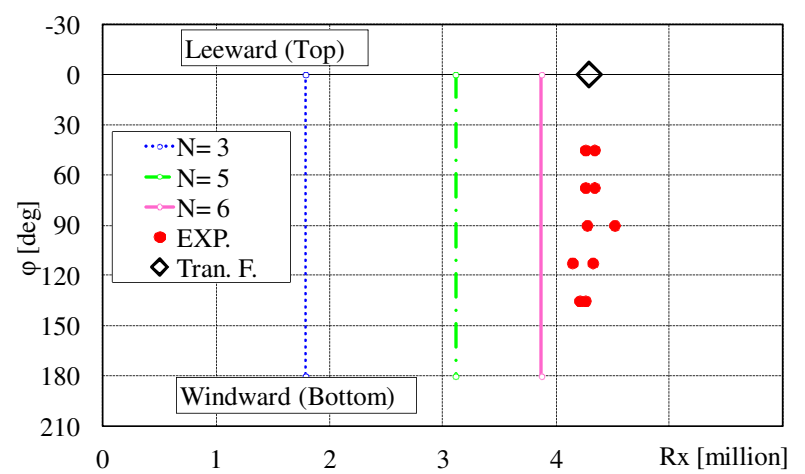

(j) Leeward plane corresponds to $\mathrm{Y}=0$ for SC-2deg- 99

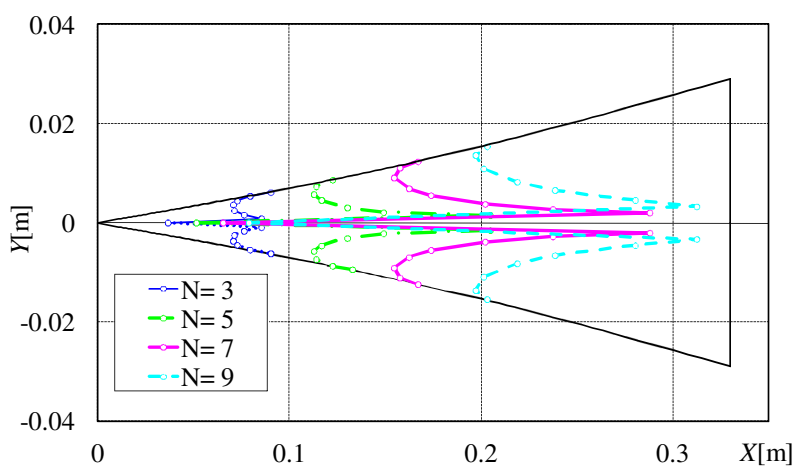

Figure 5. $N$-factor contours based on envelope method for selected test conditions (continued).

Table 2: Summary of transition locations

\begin{tabular}{|c|c|c|c|c|c|c|c|c|c|c|c|c|}
\hline \multirow[b]{2}{*}{ Shape } & \multirow[b]{2}{*}{$\begin{array}{l}\text { AOA } \\
\text { [deg] }\end{array}$} & \multirow[b]{2}{*}{$\begin{array}{c}\mathrm{P} 0 \\
{[\mathrm{kPa}]}\end{array}$} & \multicolumn{5}{|c|}{ Transition front along leeward symmetry plane } & \multicolumn{5}{|c|}{ Transition front within side region } \\
\hline & & & $\begin{array}{c}\mathrm{X} \\
{[\mathrm{m}]}\end{array}$ & $\begin{array}{c}\mathrm{Rx} \\
\text { [million] }\end{array}$ & $\begin{array}{c}\mathrm{N}- \\
\text { LSTAB }\end{array}$ & $\begin{array}{c}\mathrm{N}- \\
\text { LASTRAC }\end{array}$ & comment & $\begin{array}{c}\mathrm{X} \\
{[\mathrm{m}]}\end{array}$ & $\begin{array}{c}\mathrm{Rx} \\
{[\text { million] }}\end{array}$ & $\begin{array}{c}\varphi \\
{[\mathrm{deg}]}\end{array}$ & $\begin{array}{c}\mathrm{N}- \\
\text { LSTAB }\end{array}$ & comment \\
\hline $\mathrm{SC}$ & 0 & \multirow{5}{*}{99} & 0.33 & 4.29 & 6.2 & 5.6 & extrapolated & & & & & \\
\hline $\mathrm{SH}$ & \multirow{8}{*}{2} & & & & & & & 0.24 & 3.02 & 50 & 9.6 & - \\
\hline SSH & & & & & & & & & & & & \\
\hline SC & & & 0.14 & 1.79 & 18.4 & 16.9 & - & 0.12 & 1.51 & 35 & 5.5 & - \\
\hline FC & & & 0.11 & 1.32 & 13.5 & 10.9 & - & 0.15 & 1.80 & 30 & 6.2 & at $30 \mathrm{deg}$ \\
\hline $\mathrm{SH}$ & & \multirow{4}{*}{70} & & & & & & & & & & \\
\hline SSH & & & & & & & & 0.21 & 1.48 & 30 & 5.2 & - \\
\hline $\mathrm{SC}$ & & & 0.23 & 1.65 & 26.4 & & - & 0.19 & 1.41 & 30 & 5.1 & - \\
\hline FC & & & 0.16 & 1.14 & 13.6 & & - & 0.19 & 1.40 & 30 & 5.1 & at 30deg \\
\hline
\end{tabular}

(a) Frequency spectra of measured surface pressure fluctuations along leeward ray

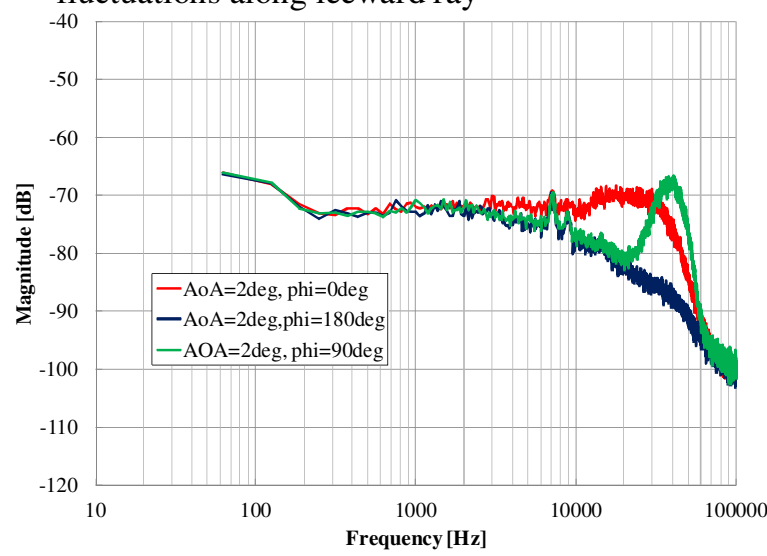

(b) Predicted $\mathrm{N}$-factors as functions of disturbance

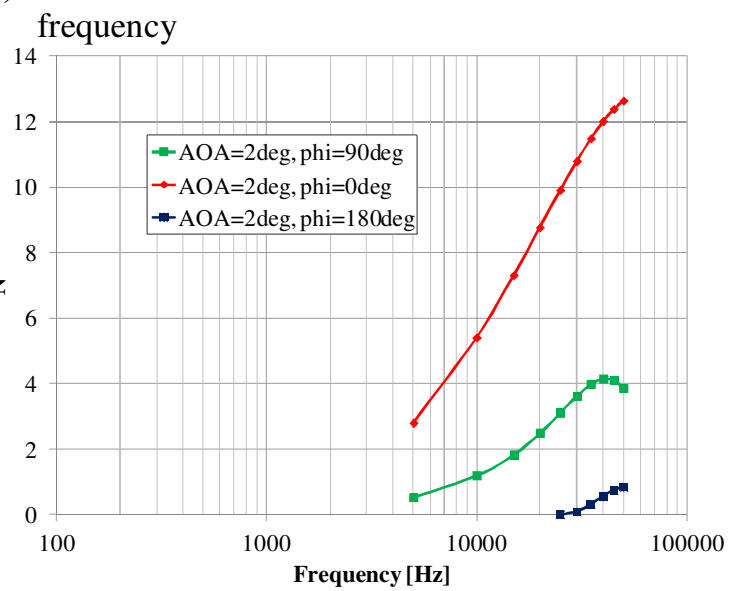

Figure 6. Comparison between frequency spectra of measuredsurface pressure fluctuations and the Nfactor predictions as a function of disturbance frequency for the SC-2deg-70 configuration. 
computational results and, therefore, is discussed later in Section V.) The transition Reynolds number of 4.29 million for the straight cone at zero angle of attack falls between the range of transition Reynolds numbers observed in previous flight experiments and conventional wind tunnel measurements for slightly higher Mach numbers ( $\mathrm{M}=2.5$ to 4.0) but similar values of unit Reynolds number. ${ }^{3,4}$ The fact that the measured transition Reynolds numbers are considerably lower than those in flight ${ }^{19}$ cannot be easily reconciled with the low values of measured free-stream pressure fluctuations in the SWT2 and FWT facilities. A more detailed study of the free-stream disturbance environment may help explain this finding.

Surface pressure fluctuations measured using the Kulite sensor can provide potentially useful information concerning boundary layer disturbances at the sensor location. The azimuthal location of the sensor could be varied, allowing one to obtain measurements at multiple values of $\varphi$. Fig. 6(a) shows the frequency spectra of surface pressure fluctuations for three different values of $\varphi$ in the case of the SC-2deg-70 configuration. The spectra for $\varphi=$ 0 deg and $\varphi=90$ deg reveal high amplitude disturbances within specific frequency bands indicating the presence of potential instability amplification. The frequencies corresponding to the spectral peaks of surface pressure fluctuation at the kulite location are approximately $20 \mathrm{kHz}$ for $\varphi=0$ deg (i.e., the leeward symmetry plane) and 40 $\mathrm{kHz}$ when $\varphi=90 \mathrm{deg}$. No significant disturbance was observed along the windward ray $(\varphi=180 \mathrm{deg})$ in this particular case. A comparison between these measured spectra and the linear stability predictions from Fig. 6(b) is postponed until Section V.

\section{Mean Flow Computations}

\section{A. Computational Methodologies}

The laminar basic state at each test condition was obtained using numerical solutions to the compressible NavierStokes equations. Due to the long duration of the tests in SWT2, the test model reaches thermal equilibrium with the surrounding flow and, hence, the thermal boundary condition at the model surface corresponds to an adiabatic wall. On the other hand, an isothermal boundary condition is more appropriate for the short duration tests corresponding to a higher stagnation pressure $\left(P_{0}=99 \mathrm{kPa}\right)$ in FWT. For the FWT test conditions, the estimated recovery temperature (based on a recovery factor of 0.85 ) is $277 \mathrm{~K}$. Thus, the isothermal model temperature of $T=$ $300 \mathrm{~K}$ used for the mean flow computations corresponds to $T_{w} / T_{a d} \approx 1.08$.

Two different flow solvers were used for this purpose and extensive comparisons were made between the respective solutions to ensure that the computed mean flow solutions were independent of the code. Computations with adiabatic thermal wall boundary conditions were performed using the 3D, multi-block, structured-grid flow solver UPACS, ${ }^{20}$ which was developed at JAXA. Independent computations for the same test conditions were performed at NASA using an analogous 3D, multi-block, structured-grid flow solver, VULCAN, ${ }^{21}$ that was developed at NASA Langley Research Center. Additional computations were done with the VULCAN code to compute the basic state solutions corresponding to the isothermal wall boundary condition $\left(T_{w}=300 \mathrm{~K}\right)$. The nose radius of each axisymmetric configuration was assumed to be zero for the UPCAS computations, whereas the VULCAN computations used a nonzero but tiny nose radius (approximately $4 \mu \mathrm{m}$ ) that was resolved with approximately 65 streamwise points in the nose region.

The UPACS based computations at JAXA were based on a typical grid size of 120 points in the streamwise direction, 150 points in the surface normal direction, and more than 193 points in the azimuthal (i.e., circumferential) direction. On the other hand, computations at NASA were based on a typical grid size of 610 points in the streamwise direction, 353 points in the surface normal direction, and more than 257 points in the azimuthal direction. The wall-normal grid distribution was similar for both flow solvers.

The grid independence of computed solutions was confirmed via preliminary comparisons between solutions on different grid sizes. To establish the grid convergence of the basic state solutions in a definitive manner, the VULCAN computations used a wider range of grid sizes, including up to 50 times larger than the grid sizes mentioned above. In order to provide sufficiently accurate description of the basic state for linear stability analysis, the UPACS grids had at least 50 to 80 grid points in the boundary layer and more than 80 points in the case of the VULCAN solutions. This type of wall-normal resolution had been found to be sufficient for linear stability analysis in a related study conducted previously. The azimuthal grids were clustered near the leeward ray in order to capture the potential influence of azimuthal diffusion on the boundary layer flow in the vicinity of the symmetry plane.

\section{B. Surface Pressure Distributions}

Figures 7(a) through 7(d) display the contours of surface pressure coefficient for each of the four body shapes at 2-degrees angle of inclidence. Regardless of the body shape, there is a negative azimuthal pressure gradient at 
any fixed axial station, which drives a circumferential flow from the windward to the leeward side and, hence, causes the boundary layer flow to become fully three-dimensional.

The pressure gradient in the axial direction varies with the body shape. As alluded to previously, it is favorable along the SH and SSH body shapes, but almost zero for the SC and adverse for the FC. The favorable pressure gradient along the length of the SSH body is weaker in comparison with that along the SH body.

(a) $\mathrm{SH}-2 \mathrm{deg}-99$

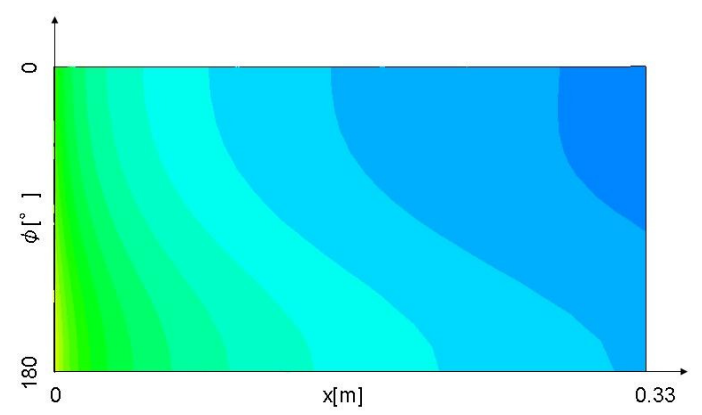

(c) SC-2deg-99

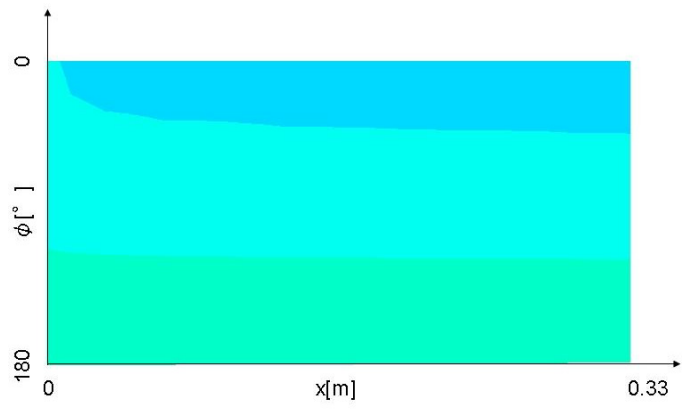

(b) SSH-2deg-99

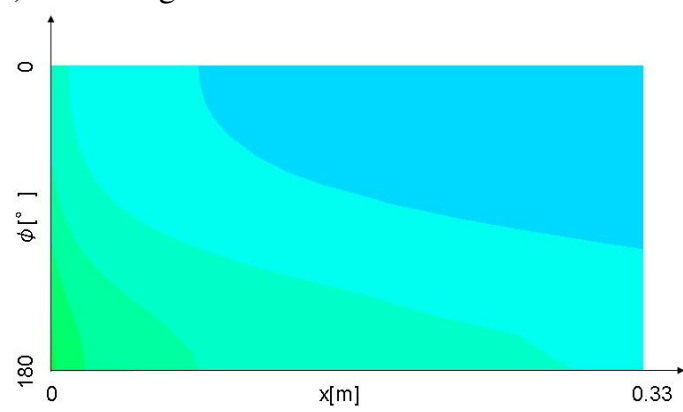

(d) FC-2deg-99

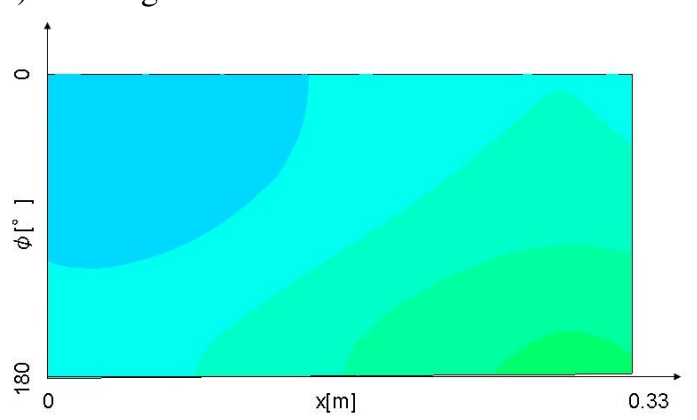

Figure 7. Surface pressure distribution at $\alpha=2$ degrees. ( $\varphi=0$ : leeward plane, $\varphi=180$ : windward plane)

\section{Mean Velocity Profiles along the Leeward Symmetry Plane}

The streamwise development of the UPACS based mean velocity profiles along the leeward ray at 2-degrees angle of incidence is shown in Figs. 8(a) through 8(d). For the purpose of comparison, velocity profiles for the axisymmetric flow over the SC body (case SC-0deg-99) are shown in Fig. 8(e). The latter set of profiles are virtually self-similar as seen from the collapse of all three curves in Fig. 8(e). In contrast, the leeward plane profiles in the SH-2deg-99 case continue to develop in the streamwise direction and are not self-similar. The variation in velocity profiles over a similar range of locations becomes stronger as the magnitude of the favorable pressure gradient in the streamwise direction is reduced from the SH body shape (Fig. 8(a)) to the SSH body shape (Fig. 8(b)). By inspecting the derivatives of the velocity profiles, it is found that the profiles downstream of $x=0.1 \mathrm{~m}$ exhibit a slight inflection. The existence of inflection is even visually obvious in the case of velocity profiles along the leeward ray of the SC-2deg-99 configuration (Fig. 8(c)) and becomes stronger when the axial pressure gradient becomes negative (case FC-2deg-99 in Fig. 8(d)). Of course, the strong effects of boundary-layer lift-up as seen from the FC-2deg-99 profile at $x=0.30113 \mathrm{~m}$ are unlikely to be observed in practice, because the boundary layer flow should transition to turbulence well ahead of this station.

\section{Comparison between computations based on UPACS and VULCAN solvers}

The laminar basic states computed with the UPACS and VULCAN flow solvers were compared with each other to establish that the boundary layer profiles used for stability analysis were independent of the flow solver and the grid topology used. To illustrate the comparison between the two sets of solutions, the streamwise variation of boundary layer thickness $\delta$, and boundary layer profiles of streamwise velocity and temperature at select stations within the leeward symmetry plane are shown in Figs. 9(a)-(c), respectively. Here, the boundary layer thickness is based on the location where the relation $[d(\rho U) / d y]_{\delta}=0.01 \times[d(\rho U) / d y]_{\text {wall }}$ is satisfied. 
Fig. 9(a) shows that the boundary layer thicknesses obtained from the UPACS and VULCAN solutions for the adiabatic surface condition agree very well with each other. Furthermore, the comparison between the VULCAN solutions for adiabatic and isothermal surface conditions indicates that the effect of thermal boundary condition on boundary layer thickness is small. Although omitted from this paper, other quantities at the boundary layer edge, such as the edge pressure and edge density distribution along the body length are also in good agreement among the three solutions (namely, adiabatic case with UPACS and VULCAN, and isothermal case with VULCAN). The wallnormal profiles of temperature and density as obtained from the UPACS and VULCAN computations for the adiabatic wall case are also in agreement with each other; however, as may be expected, these two sets of profiles show visible differences from those corresponding to the isothermal surface (Fig. 9(c)). On the other hand, the profiles of streamwise velocity based on all three solutions show relatively small differences (Fig. 9(b)), indicating that the velocity profiles are less sensitive to the thermal boundary condition at the surface. Even smaller differences are seen between the velocity profiles based on the UPACS and VULCAN solutions for an adiabatic wall. Since grid convergence of the VULCAN solutions had been established, these very small differences between the VULCAN AND UPACS solutions for an adiabatic wall are attributed to slight shortcomings of grid resolution in the UPACS solution; furthermore, these differences were noticeable only in the vicinity of the leeward plane and not over the rest of the cone.

\section{Linear Stability Analysis and Comparison with Measurements}

The laminar mean flows described in the previous section were used as basic states for linear stability analysis to understand the measured transition characteristics near the leeward ray. Computations were performed using the LSTAB $^{6}$ code developed at JAXA and the LASTRAC ${ }^{22}$ code developed at NASA Langley Research Center. A selected set of results based on the $\mathrm{e}^{N}$ methodology is described below.

\section{A. Envelope Method}

The envelope method correlates the transition location with the logarithmic amplification ratio (i.e., N-factor) based on the most amplified fixed frequency disturbances. For each frequency, the $\mathrm{N}$-factor distribution over the body surface is determined by integrating the maximum growth rate over all azimuthal wave numbers at each point along a selected set of trajectories. In this paper, these trajectories are taken to be streamlines near the edge of the boundary layer. $\mathrm{N}$-factor contours corresponding to LSTAB computations based on the UPACS mean flow solutions for 2-degree angle of incidence and both values of stagnation pressure in Table $1\left(\mathrm{P}_{0}=99 \mathrm{kPa}\right.$ and $\left.\mathrm{P}_{0}=70 \mathrm{kPa}\right)$ were included in Figs. 5(a) through 5(h), which were partially discussed in Section III in the context of the experimentally inferred transition fronts. For the purpose of comparison, results pertaining to axisymmetric flow over the SC body are shown in Fig. 5(i). As expected, an azimuthally invariant transition front is predicted in the last case. To enable an easier visual comparison between the experimentally measured heat transfer distributions in Fig. 2 and the Nfactor contours for a selected configuration, the top view of the N-factor contours for the SC-2deg-99 configuration is shown in Fig. 5(j), wherein the the horizontal axis $(Y=0)$ corresponds to the leeward symmetry plane.

$\mathrm{N}$-factor contours for the SH body predict a later transition location along the leeward plane than the neighboring azimuthal locations, regardless of stagnation pressure $P_{0}$ (Fig. 5(a),(b)). In contrast, computations for the other 3 body shapes (Figs. 5(b) through 5(h)) predict a 3-lobed transition front including a center lobe that is indicative of an earlier transition location along the leeward ray in comparison with the adjacent azimuthal locations. The center lobe moves progressively farther upstream as the streamwise pressure gradient changes from mildly favorable (SSH) to nearly zero (SC) to adverse (FC). This upstream movement of transition location along the leeward ray is attributed to the higher amplification rates associated with increasingly thicker, and highly inflectional velocity profiles along the leeward plane (Fig. 8). This predicted movement is also consistent with the measured trend in transition location along the leeward ray as a function of the body shape as described earlier in Fig. 2.

As shown in Fig. 5(e), the earliest transition in the SC-2deg-99 case is predicted to occur along the leeward ray $(\varphi=0)$ rather than along the adjoining side surface. The integration trajectories used for $\mathrm{N}$-factor calculations in this case are also shown in the same figure. The apex (i.e., most upstream location) of the crossflow dominated transition lobes on the side of the cone was found to be located at approximately $\varphi=60$ degrees, in agreement with a previous prediction for the same cone at the same Mach number. ${ }^{6,8}$ The farthest downstream onset of transition is predicted to occur along the windward ray, i.e., at $\varphi=180$ degrees and this location is farther downstream than the predicted transition for a zero angle of attack case (Fig. 5(i)).

The predicted occurrence of a middle lobe in the transition front over the SC and FC bodies and its absence over the $\mathrm{SSH}$ body at $P_{0}=99 \mathrm{kPa}$ is in agreement with the experimentally observed transition fronts as shown in Fig. 2 . However, while the measured transition front for the SSH body predicted later transition along the leeward ray, the 
corresponding $\mathrm{N}$-factor contours do predict a local minimum in the transition location along the leeward ray. This discrepancy could perhaps be explained if the $\mathrm{N}$-factor value correlating with crossflow induced transition over the side region is sufficiently smaller than the $\mathrm{N}$-factor value correlating with transition due to first mode instability along the leeward ray. Such differences in $\mathrm{N}$-factor correlations are easily possible due to differences in receptivity characteristics as well as nonlinear mechanisms related to the respective underlying instability mechanisms. An additional difference between the predicted $\mathrm{N}$-factor contours and the measured transition fronts corresponds to the weaker signature of the outer (crossflow) lobe in the measured transition front over the FC (Figs. 2(g) and 2(h)), such that there is a nearly monotonic downstream shift in the measured transition location at increasing azimuthal angles from the leeward ray. This might have happened because the boundary layer flow along the azimuthal orientations corresponding to the inner portions of the crossflow transition lobes transitioned further upstream due to turbulent contamination from the earlier transition location along the leeward ray.

The numerically predicted $\mathrm{N}$-factor values at the measured transition locations along the leeward ray and the farthest upstream location of the crossflow transition lobe (see Fig. 5) for the various flow configurations are summarized in Table 2. It may be observed that the $\mathrm{N}$-factors at the transition location along the leeward ray are always greater than 13.5 and, hence, are much higher than the $\mathrm{N}$-factor value of 6.2 correlating with measured transition under axisymmetric conditions. The latter value is consistent with the expectation that the correlating Nfactor for transition in axisymmetric flow over a cone would be significantly smaller than the range of $\mathrm{N}$-factor values $(\mathrm{N}=9$ to 10$)$ correlating with transition measurements for similar flows in a quiet tunnel. ${ }^{4}$ The increase in $\mathrm{N}$ factor for transition along the leeward ray is much too large to be explained by the fact that the measured transition location is based on the middle of transition zone rather than with the transition onset location (which is only about 10 percent upstream compared to the midpoint of transition zone).

The reason for the extraordinarily high $\mathrm{N}$-factors may be related to certain discrepancies between the computed and measured boundary layer profiles (not shown in this paper). This conjecture does not reflect a lack of accuracy in the numerical computation, but a lack of sufficient information to model the flow distortions associated with the imperfections of the model tip and/or flow quality effects such as flow angularity, etc. It is also possible that the spanwise gradients of the basic state, although zero along the leeward ray, become large enough in the immediate vicinity of the leeward plane to influence the disturbance evolution within the leeward plane. Furthermore, the receptivity mechanisms associated with the leeward flow could be weaker than those of the axisymmetric boundary layer, perhaps because of the somewhat higher frequencies of the instability modes, which could cause a delay in transition and lead to the higher $\mathrm{N}$ factors.

The $\mathrm{N}$-factor values at the apex of the experimentally inferred transition lobe on the side of the cone models are much lower than those along the leeward symmetry plane but are comparable to the $\mathrm{N}$-factor at the measured transition location for first mode transition in the axisymmetric case SC-0deg-99 (Figure 10). The low N-factor values in the axisymmetric case as well as for the side lobe at the nonzero angle of attack are comparable to the Nfactor values correlating with previous transition measurements in conventional (i.e., noisy) facilities, in spite of the fact that the measured levels of free-stream acoustic fluctuations in the FWT and SWT2 facilities appear to be much lower than other conventional facilities. The reasons behind the low $\mathrm{N}$ factors may be related to inaccuracies in determining transition locations from the IR measurement, imperfections in model geometry (anisotropy of nose tip, curvature discontinuity, small scale perturbations in surface geometry) and the potential presence of non-acoustic free-stream disturbances.

The most amplified frequency predicted by the stability analysis was compared with the frequency spectrum of the experimentally observed surface pressure fluctuations for the SC-2deg-70 configuration (Fig. 6). According to the envelope method, the most amplified disturbance at the kulite location had a frequency of more than $50 \mathrm{kHz}$ along the leeward and windward rays and about $40 \mathrm{kHz}$ at $\varphi=90 \mathrm{deg}$. This prediction is in fairly in good agreement with the measured spectrum at $\varphi=90 \mathrm{deg}$, but larger than the measured peak along the leeward ray. Along the windward ray, the disturbance amplitudes in the experiment are too small to be compared with theoretical predictions. The reason for the discrepancy along the leeward ray could not be determined. Even though the computed mean flow profiles were shown to be grid converged, at attempt to measure the boundary layer profiles along the leeward ray did reveal some differences from the computed mean flow profile. However, whether or not those differences would have accounted for most of the observed discrepancies remains an open question. Other reasons potentially contributing to this uncertainty were outlined in the context of the large values of $\mathrm{N}$-factors along the leeward ray.

\section{B. Mode Tracking Method}

An alternative to the envelope method for $\mathrm{N}$-factor correlations is the mode tracking method. Rather than maximizing the local growth rate over all disturbance wave numbers at a given frequency, the mode tracking 
method uses the envelope of several $\mathrm{N}$-factor curves, each of which corresponds to a nominally fixed disturbance entity in the form of a specified set of frequency-wave number combination. Stability computations using the mode tracking approach were performed using the LASTRAC code. An illustrative comparison of $\mathrm{N}$-factor contours based on the mode tracking method with those obtained with the envelope method is shown in Fig. 10. This comparison is made for the SC-2deg-99 configuration using basic states obtained for an adiabatic model surface; however, results of mode tracking analysis based on the isothermal condition $\left(T_{w} / T_{a d} \approx 1.08\right)$ are also included. The basic states for the mode tracking (LASTRAC) and envelope (LSTAB) analyses were obtained using two different mean flow solvers (VULCAN and UPACS, respectively) as described previously. However, the mean flow profiles predicted by VULCAN and UPACS were in close agreement with each other, and hence the results in Fig. 10 may be assumed to represent the effects of the different methods of $\mathrm{N}$-factor computation and, to a lesser degree, different stability codes used for the $\mathrm{N}$-factor calculation.

(a) $\mathrm{SH}-2$ deg-99

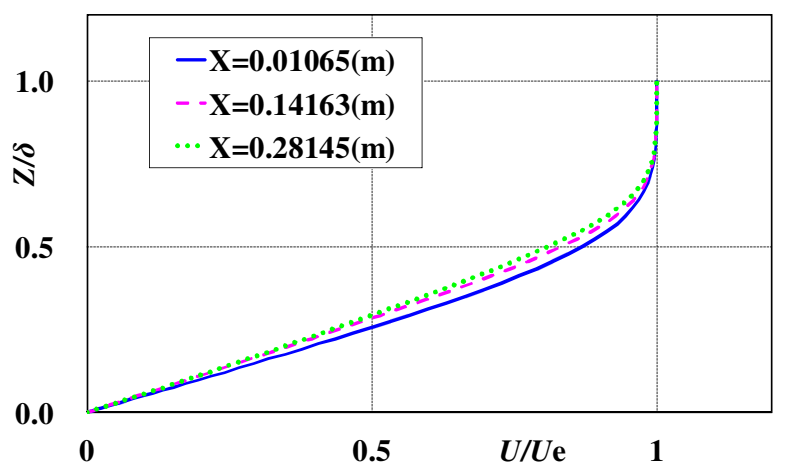

(c) SC-2deg-99

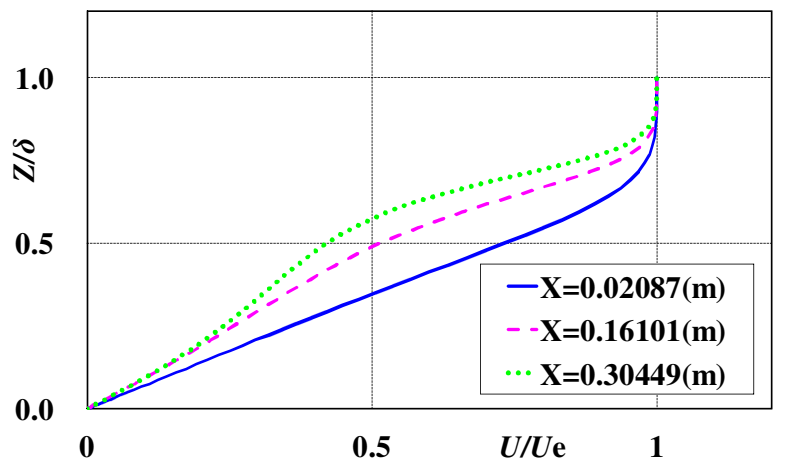

(b) SSH-2deg-99

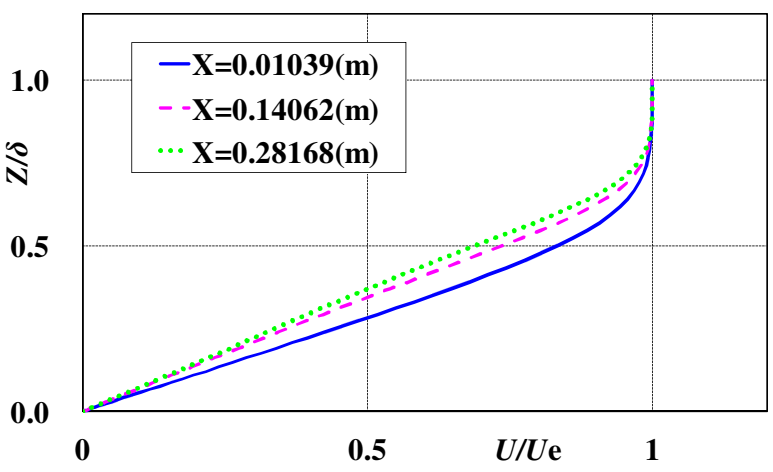

(d) FC-2deg-99

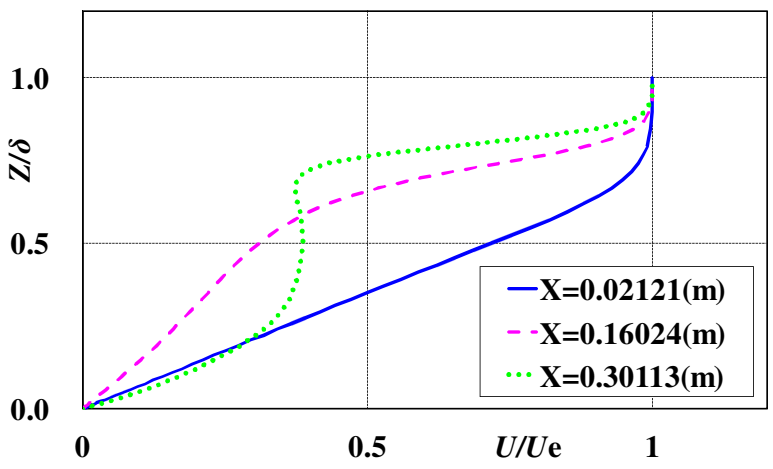

(e) SC-0deg-99

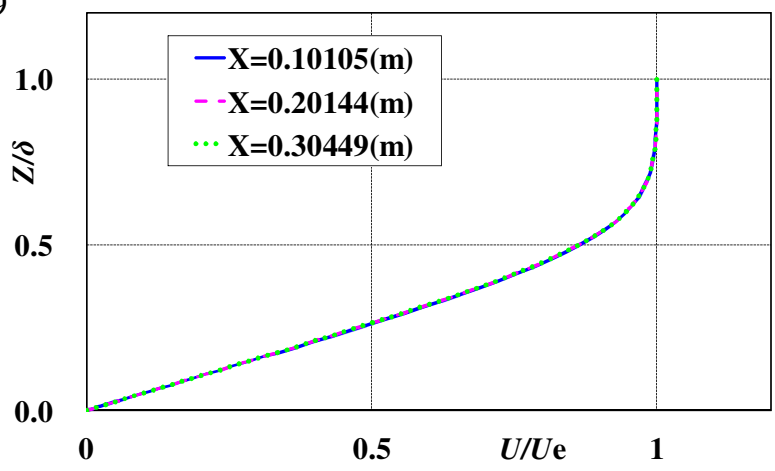

Figure 8. Mean velocity profiles along the leeward symmetry plane. 
(a) boundary layer thickness $\delta$

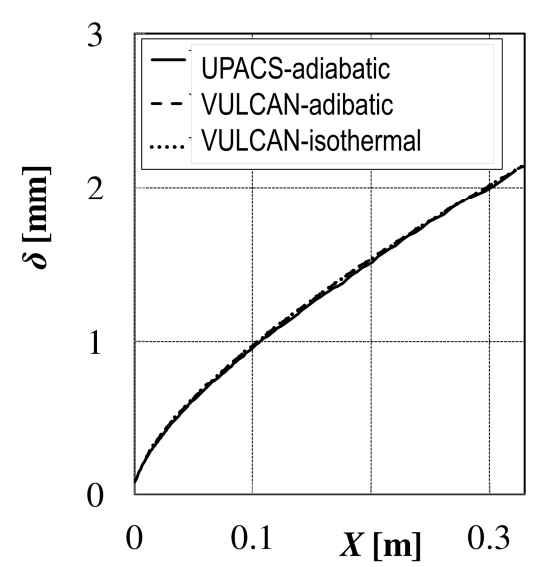

(b) boundary layer profiles of streamwise velocity

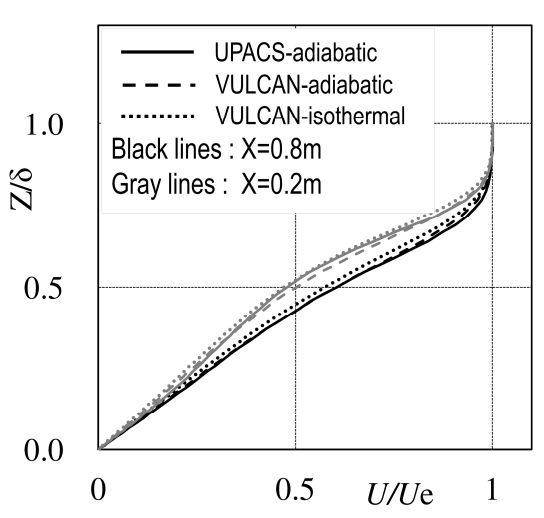

(c) boundary layer profiles of temperature

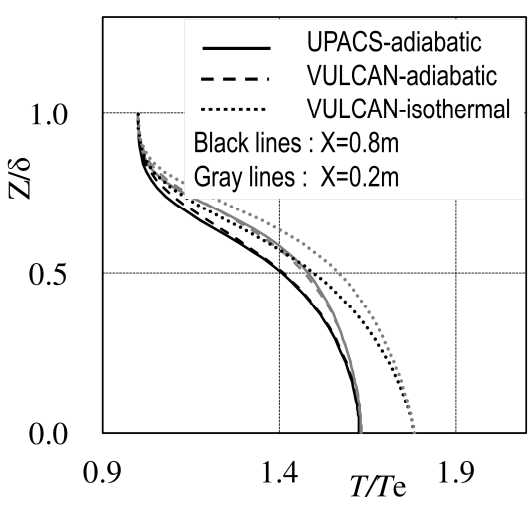

Figure 9. Comparison between UPACS and VULCAN based predictions of mean velocity characteristics along the leeward symmetry plane for SC-2deg-99 case.

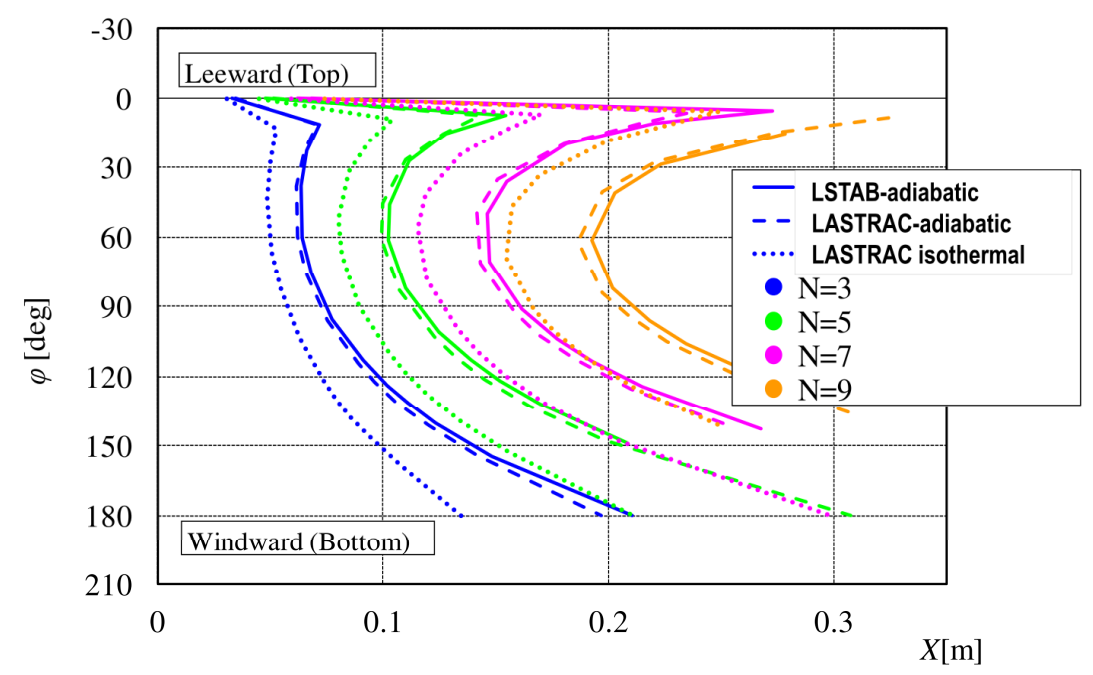

Figure 10. Comparison between results of stability analysis for SC-2deg-99 configuration.

Fig. 10 shows that, for the SC-2deg-99 configuration at least, the N-factor contours predicted by the envelope method are similar to those predicted by the mode tracking method, but some quantitative difeerences remain because of the different assumptions in computing the $\mathrm{N}$-factor envelope. Furthermore, the thermal boundary condition also has a visible impact on the $\mathrm{N}$-factor contours, especially away from the leeward plane of symmetry. The upstream shift in $\mathrm{N}$-factor contours for the isothermal case reflects the destabilizing effect of increased wall temperature on both first mode and crossflow instabilities. Effectively, the isothermal condition leads to an increase in $\mathrm{N}$-factor of between 1 and 2 in this case.

\section{Summary and Concluding Remarks}

Boundary layer transition along the leeward symmetry plane of axisymmetric bodies at a non-zero angle of incidence in supersonic freestream was investigated via conventional facility experiments and numerical computations as part of joint research between JAXA and NASA. Transition over four axisymmetric bodies (namely, Sears-Haack body, semi-Sears-Haack body, straight cone and flared cone) with different streamwise pressure gradients was studied. Experimental measurements included visualization of transition front via heat transfer distributions inferred from the surface temperature measurements using an IR camera, along with limited 
measurements of surface pressure fluctuations and mean boundary layer profiles along the leeward symmetry plane. The measurements indicate that the boundary layer flow along the leeward symmetry plane may transition earlier than that along the neighboring azimuthal locations when the streamwise pressure gradient is zero or adverse. The earlier transition along the leeward ray under non-favorable axial pressure gradients is consistent with the computational predictions which indicate increasingly thicker and more strongly inflectional (and correspondingly more unstable) boundary layer profiles along the leeward symmetry plane as the streamwise pressure gradient becomes relatively more adverse. The destabilizing effect of pressure gradient on the boundary layer flow within the leeward plane is analogous to that in purely two-dimensional (or axisymmetric) boundary layer flows. However, in the present context, it is caused by the three-dimensional dynamics associated with increasing build-up of secondary flow along the leeward plane under an adverse axial pressure gradient. In other words, the same configurations do not display such dramatic effect of the body shape on boundary layer stability under zero-angleof-attack conditions. Computations also confirm the weakened instability of the boundary layer flow along the leeward symmetry plane under a sufficiently strong favorable pressure gradient. Additional analysis is necessary to establish whether turbulent contamination from the adjacent region of crossflow dominated transition might play a role during transition along the leeward plane under favorable pressure gradient.

To our knowledge, this paper provides the first IR based global measurements of boundary layer transition over a yawed circular cone in the supersonic regime, albeit with a significant uncertainty in the quantitative data pertaining to transition locations. More significantly, the profound effect of axial pressure gradient on transition behavior along the leeward symmetry plane of slender axisymmetric bodies at a nonzero angle of incidence has been demonstrated for the first time.

The present work has also identified certain discrepancies between the predicted $\mathrm{N}$-factor contours and the experimentally observed transition fronts for certain cases, as well as indicating rather high $\mathrm{N}$-factor values for leeward transition even in a conventional facility These discrepancies indicate the potential sensitivity of boundary layer transition along the leeward symmetry plane to external disturbances and/or turbulent contamination and thereby underscore the limitations of $\mathrm{N}$-factor correlations for boundary layer flow involving multiple instability mechanisms. The need to perform more detailed measurements of leeward line transition, both in terms of basic state profiles and the boundary layer perturbations, especially in a low disturbance environment, is also highlighted by the present findings. Resolving these discrepancies and addressing the need for more definitive measurements are important topics for future work and are likely to be targeted in the course of future experiments in NASA's supersonic low disturbance tunnel.

\section{Acknowledgments}

The JAXA authors would like to thank Dr. K. Yoshida for his valuable advice. They also acknowledge the support for experiments from Mr. H. Sugiura, Dr. S. Koike, and Dr. K. Nakakita of JAXA, along with Mr. T. Murayama, Mr. A. Nose, and Mr. K. Fujisaki from Gakushuin University. The work at NASA was performed as part of the Supersonic Cruise Efficiency -Airframe discipline of the Supersonics Project of NASA's Fundamental Aeronautics Program (FAP). Technical discussions with Dr. M. Malik, Dr. R. Rubinstein, Ms. E. Lee-Rausch, Mr. S. Wilkinson, Mr. L. Owens, and Dr. P. Balakumar at NASA Langley Research Center are also acknowledged.

\section{References}

1. Vijgen, P. M. H. W. and Holmes, B. J., "Experimental and Numerical Analyses of Laminar Boundary-Layer Flow Stability over an Aircraft Fuselage Forebody," Research in Natural Laminar Flow and Laminar-Flow Control, Part 3, NASA CP-2487, 1987, pp. 861-886.

2. Kroo, I., "Unconventional Configurations for Efficient Supersonic Flight," VKI lecture series on Innovative Configurations and Advanced Concepts for Future Civil Aircraft, June 6-10, 2005.

3. Dougherty, N. S. and Fisher, D. F., "Boundary Layer Transition on a 10-degree Cone:Wind Tunnel/Flight Data Correlation," AIAA Paper 80-1054, 1980.

4. Chen F.-J., Malik, M. R., and Beckwith, I. E., "Boundary-Layer Transition on a Cone and Flat Plate at Mach 3.5," AIAA Journal, Vol. 27, No. 6, 1989, pp. 687-693.

5. King, R. A., "Three-Dimensional Boundary-Layer Transition on a Cone at Mach 3.5," Experiments in Fluids, Vol. 13, 1992, pp. 305-314.

6. Ueda, Y., Ishikawa, H., and Yoshida, K., "Three Dimensional Boundary Layer Transition Analysis in Supersonic Flow Using a Navier-Stokes Code," Proceedings of International Congress of the Aeronautical Science 2004-2.8.2, [CD-ROM], 2004.

7. Lin T. C. and Rubin, S. G., "Viscous Flow over a Cone at Moderate Incidence. Part 2. Supersonic Boundary Layer," J. Fluid Mech., Vol. 59, 1973, pp. 593-620.

8. Malik, M. and Balakumar, P., "Instability and Transition in Three Dimensional Supersonic Boundary Layers," AIAA Paper 92-5049, 1992. 
9. Murayama, T., Nose, A., Tokugawa, N., Ishikawa, H., and Fujisaki, K., "Compressive Boundary-Layer Transition near the Top-Line of an Axisymmetric Body at Incidence," Proceedings of 2007 Annual Meeting, Japan Society of Fluid Mechanics, [CD-ROM], 2007 (in Japanese).

10. Sugiura, H., Tokugawa, N., Nishizawa, A., Ueda, Y., Ishikawa, H., and Yoshida, K., "Boundary-Layer Transition on Axisymmetric Bodies with Angles of Attack in Supersonic Flow," Proceedings of 2003 Annual Meeting, Japan Society of Fluid Mechanics, pp.352-353, 2003 (in Japanese).

${ }^{11 .}$ Cattafesta, L. N., III, Iyer, V., Masad, J. A, King, R. A., and Dagenhart, J. R., "Three-Dimensional Boundary-Layer Transition on a Swept Wing at Mach 3.5," AIAA Journal, Vol. 33, No. 11, 1995, pp. 2032-2037.

12. Berger, K., Rufer, S., Kimmel, R., and Adamczak, D., "Aerothermodynamic Characteristics of Boundary Layer Transition and Trip Effectiveness of the HIFiRE Flight 5 Vehicle," AIAA Paper 2009-4055, 2009.

${ }^{13 .}$ Choudhari, M., Chang, C.-L., Jentink, T., Li, F., Berger, K., Candler, G., and Kimmel, R. L., "Transition Analysis for the HIFiRE-5 Vehicle," AIAA Paper 2009-4056, 2009.

14. Owens, L., Kegerise, M., and Wilkinson, S. "Off-Body Boundary-Layer Measurement Techniques Development for Supersonic Low-Disturbance Flows,” AIAA Paper 2011-284, 2011.

15. Nose, A., Ishikawa, H., Ueda, Y., Murayama, T., and Tokugawa, N., "Influence of the Pressure Gradient on Compressive Boundary-Layer Transition on an Axisymmetric Body at Incidence," Proceedings of 2007 Annual Meeting, Japan Society of Fluid Mechanics, [CD-ROM], 2007 (in Japanese).

16. Sawada, H., Kohno, T., and Kunimasu, T., "Pressure Fluctuation Measurements in the NAL 0.2-m Supersonic Wind Tunnel," AIAA Paper 98-0636, 1998.

17. Cook, W. J., and Felderman, E .J. , "Reduction of Data from Thin-Film Heat-Transfer Gages: A Concise Numerical Technique," AIAA J., Vol.4, No.3, 1966, pp.561-562.

18. Carpenter, A., Saric, W. S., and Reed, H. L., "Laminar Flow Control on a Swept Wing with Distributed Roughness," AIAA Paper 2008-7335, 2008.

19. Dougherty, N. S., Jr., and Fisher, D. F., "Boundary Layer Transition Correlation on a Slender Cone in Wind Tunnels and Flight for Indications of Flow Quality," AEDC TR-81-26, Feb. 1982.

20. Yamazaki, H., Enomoto, S. and Yamamoto, K., “A Common CFD Platform UPCAS," High Performance Computing, Lecture Notes in Computer Science, Vol. 1940, 2000, pp. 182-190.

${ }^{21 .}$ Litton, D. K., Edwards, J. R. and White, J. A., "Algorithmic Enhancements to the VULCAN Navier-Stokes Solver," AIAA 2003-3979, June, 2003. (Also: http://vulcan-cfd.larc.nasa.gov (Aug. 18, 2011))

22. Chang, C.-L., "LASTRAC.3d: Transition Prediction in 3D Boundary Layers," AIAA Paper 2004-2542, 2004. 